\title{
A UNIFORMLY ACCURATE MULTISCALE TIME INTEGRATOR PSEUDOSPECTRAL METHOD FOR THE KLEIN-GORDON EQUATION IN THE NONRELATIVISTIC LIMIT REGIME*
}

\author{
WEIZHU $\mathrm{BAO}^{\dagger}, \mathrm{YONGYONG} \mathrm{CAI}^{\ddagger}$, AND XIAOFEI ZHAO ${ }^{\dagger}$
}

\begin{abstract}
We propose and analyze a multiscale time integrator Fourier pseudospectral (MTIFP) method for solving the Klein-Gordon (KG) equation with a dimensionless parameter $0<\varepsilon \leq 1$ which is inversely proportional to the speed of light. In the nonrelativistic limit regime, i.e. $0<\varepsilon \ll 1$, the solution of the KG equation propagates waves with amplitude at $O(1)$ and wavelength at $O\left(\varepsilon^{2}\right)$ in time and $O(1)$ in space, which causes significantly numerical burdens due to the high oscillation in time. The MTI-FP method is designed by adapting a multiscale decomposition by frequency (MDF) to the solution at each time step and applying an exponential wave integrator to the nonlinear Schrödinger equation with wave operator under well-prepared initial data for $\varepsilon^{2}$-frequency and $O(1)$ amplitude waves and a KG-type equation with small initial data for the reminder waves in the MDF. We rigorously establish two independent error bounds in $H^{2}$-norm to the MTI-FP method at $O\left(h^{m_{0}}+\tau^{2}+\varepsilon^{2}\right)$ and $O\left(h^{m_{0}}+\tau^{2} / \varepsilon^{2}\right)$ with $h$ mesh size, $\tau$ time step and $m_{0} \geq 2$ an integer depending on the regularity of the solution, which immediately imply that the MTI-FP converges uniformly and optimally in space with exponential convergence rate if the solution is smooth, and uniformly in time with linear convergence rate at $O(\tau)$ for all $\varepsilon \in(0,1]$ and optimally with quadratic convergence rate at $O\left(\tau^{2}\right)$ in the regimes when either $\varepsilon=O(1)$ or $0<\varepsilon \leq \tau$. Numerical results are reported to confirm the error bounds and demonstrate the efficiency and accuracy of the MTI-FP method for the KG equation, especially in the nonrelativistic limit regime.
\end{abstract}

Key words. Klein-Gordon equation, nonrelativistic limit, multiscale decomposition, multiscale time integrator, uniformly accurate, meshing strategy, exponential wave integrator, spectral method

AMS subject classifications. 65M12, 65M15, 65M70, 81Q05

1. Introduction. In this paper, we consider the dimensionless Klein-Gordon (KG) equation in $d$ dimensions $(d=1,2,3)$ [5, 15, 18, 19, 27, 30, 34, 39, 40]:

$$
\begin{cases}\varepsilon^{2} \partial_{t t} u-\Delta u+\frac{1}{\varepsilon^{2}} u+f(u)=0, \quad \mathbf{x} \in \mathbb{R}^{d}, & t>0, \\ u(\mathbf{x}, 0)=\phi_{1}(\mathbf{x}), & \partial_{t} u(\mathbf{x}, 0)=\frac{1}{\varepsilon^{2}} \phi_{2}(\mathbf{x}), \quad \mathbf{x} \in \mathbb{R}^{d} .\end{cases}
$$

Here $t$ is time, $\mathbf{x}$ is the spatial coordinate, $u:=u(\mathbf{x}, t)$ is a complex-valued scalar field, $0<\varepsilon \leq 1$ is a dimensionless parameter which is inversely proportional to the speed of light, $\phi_{1}$ and $\phi_{2}$ are two given complex-valued initial data which are independent of $\varepsilon$, and $f(u): \mathbb{C} \rightarrow \mathbb{C}$ is a given gauge invariant nonlinearity which is independent of $\varepsilon$ and satisfies $[6,15,28,30,34$ ]

$$
f\left(e^{i s} u\right)=e^{i s} f(u), \quad \forall s \in \mathbb{R} .
$$

We remak that when the initial data $\phi_{1}(\mathbf{x}), \phi_{2}(\mathbf{x}): \mathbb{R}^{d} \rightarrow \mathbb{R}$ and $\mathbf{f}(u): \mathbb{R} \rightarrow \mathbb{R}$, then the solution $u(\mathbf{x}, t)$ of (1.1) is real-valued. In this case, the gauge invariant condition (1.2) for the nonlinearity in (1.1) is no longer needed. Thus (1.1) includes the classical KG equation with the solution $u$ real-valued as a special case [7, 14, 31, 36, 37, 39, 40].

\footnotetext{
* This research was supported by the Singapore A*STAR SERC psf-grant 1321202067.

${ }^{\dagger}$ Department of Mathematics, National University of Singapore, Singapore 119076 (matbaowz@nus.edu.sg, http://www.math.nus.edu.sg/ bao/; zhxfnus@gmail.com).

$\ddagger$ Department of Mathematics, Purdue University, West Lafayette, IN 47907, USA (cai99@math.purdue.edu).
} 
The above KG equation is also known as the relativistic version of the Schrödinger equation used to describe the dynamics of a spinless particle [34. In most applications [15, 18, 21, 27, 29, 30, 33, $f(u)$ is taken as the pure power nonlinearity, i.e.

$$
f(u)=g\left(|u|^{2}\right) u, \text { with } g(\rho)=\lambda \rho^{p} \text { for some } \lambda \in \mathbb{R}, p \in \mathbb{N}_{0}:=\mathbb{N} \cup\{0\},
$$

and then the KG equation (1.1) conserves the energy [5, 18, 19, 30]

$$
\begin{aligned}
E(t) & :=\int_{\mathbb{R}^{d}}\left[\varepsilon^{2}\left|\partial_{t} u(\mathbf{x}, t)\right|^{2}+|\nabla u(\mathbf{x}, t)|^{2}+\frac{1}{\varepsilon^{2}}|u(\mathbf{x}, t)|^{2}+F\left(|u(\mathbf{x}, t)|^{2}\right)\right] d \mathbf{x} \\
(1.4) & \equiv \int_{\mathbb{R}^{d}}\left[\frac{1}{\varepsilon^{2}}\left|\phi_{2}(\mathbf{x})\right|^{2}+\left|\nabla \phi_{1}(\mathbf{x})\right|^{2}+\frac{1}{\varepsilon^{2}}\left|\phi_{1}(\mathbf{x})\right|^{2}+F\left(\left|\phi_{1}(\mathbf{x})\right|^{2}\right)\right] d \mathbf{x}=E(0), t \geq 0
\end{aligned}
$$

where $F(\rho)=\int_{0}^{\rho} g(s) d s$.

For a fixed $\varepsilon=\varepsilon_{0}=O(1)$, i.e. $\mathrm{O}(1)$-speed of light regime (e.g. $\varepsilon=1$ ), the KG equation (1.1) has been studied extensively in both analytical and numerical aspects, see [7, 10, 14, 18, 21, 26, 31, 33, 36, 37, 39, 40, and references therein. Recently, more attentions have been devoted to analyzing the solution structure $[28,30,32,41]$ and designing efficient and accurate numerical methods [5, 15] of the problem (1.1) in the nonrelativistic limit regime, i.e. $0<\varepsilon \ll 1$. In fact, due to that the energy $E(t)=O\left(\varepsilon^{-2}\right)$ in (1.4) becomes unbounded when $\varepsilon \rightarrow 0$, this brings significant difficulties in the mathematical analysis of the problem (1.1) in the nonrelativistic limit regime. Based on recent analytical results [28,30,32,41], the problem (1.1) propagates waves with amplitude at $O(1)$ and wavelength at $O\left(\varepsilon^{2}\right)$ and $O(1)$ in time and space, respectively, when $0<\varepsilon \ll 1$. To illustrate this, Fig. 1.1 shows the solution of the KG equation (1.1) with $d=1, f(u)=|u|^{2} u, \phi_{1}(x)=e^{-x^{2} / 2}$ and $\phi_{2}(x)=\frac{3}{2} \phi_{1}(x)$ for different $\varepsilon$. This highly oscillatory nature of the solution of (1.1) causes severe burdens in practical computation, making the numerical approximation of (1.1) extremely challenging and costly in the regime of $0<\varepsilon \ll 1$. Different numerical methods, including finite difference time domain (FDTD) methods [5], exponential wave integrator Fourier pseudospectral (EWI-FP) method [5] and asymptotic preserving (AP) method [15] have been proposed and analyzed as well as compared for solving (1.1) numerically in the nonrelativistic limit regime. In the above numerical study, they paid particular attention on the resolution of different numerical methods, i.e. meshing strategy requirement (or $\varepsilon$-scalability) for (1.1) when $0<\varepsilon \ll 1$. Based on their results, in order to capture 'correctly' the oscillatory solution of (1.1) in practical computations, the frequently used FDTD methods request mesh size $h=O(1)$ and time step $\tau=O\left(\varepsilon^{3}\right)$ and the EWI-FP methods require $h=O(1)$ and $\tau=O\left(\varepsilon^{2}\right)$, when $0<\varepsilon \ll 1[5]$. Thus the FDTD and EWI-FP methods converges optimally in space and time for any fixed $\varepsilon=\varepsilon_{0}=O(1)$, but they do not converge when $\tau=O(\varepsilon)$. Similarly, the AP method [15] requests $h=O(1)$ and $\tau=O(1)$ when $0<\varepsilon \ll 1$, but it brings $O(1)$-error when $\varepsilon=\varepsilon_{0}=O(1)$. Thus all the above numerical methods for the problem (1.1) do not converge uniformity for $\varepsilon \in(0,1]$ [5, 15]. Recently, uniformly accurate numerical schemes for high oscillatory Klein-Gordon and nonlinear Schrödinger equations have been proposed and analyzed [8] based on embedding the problem in a suitable "two-scale" reformulation with the induction of an additional variable and using the Chapman-Enskog expansion to separate the fast time scale and the slow one.

Very recently, by using a highly oscillatory second-order ordinary differential equation (ODE) which has the same oscillatory nature as the problem (1.1) in time, we 

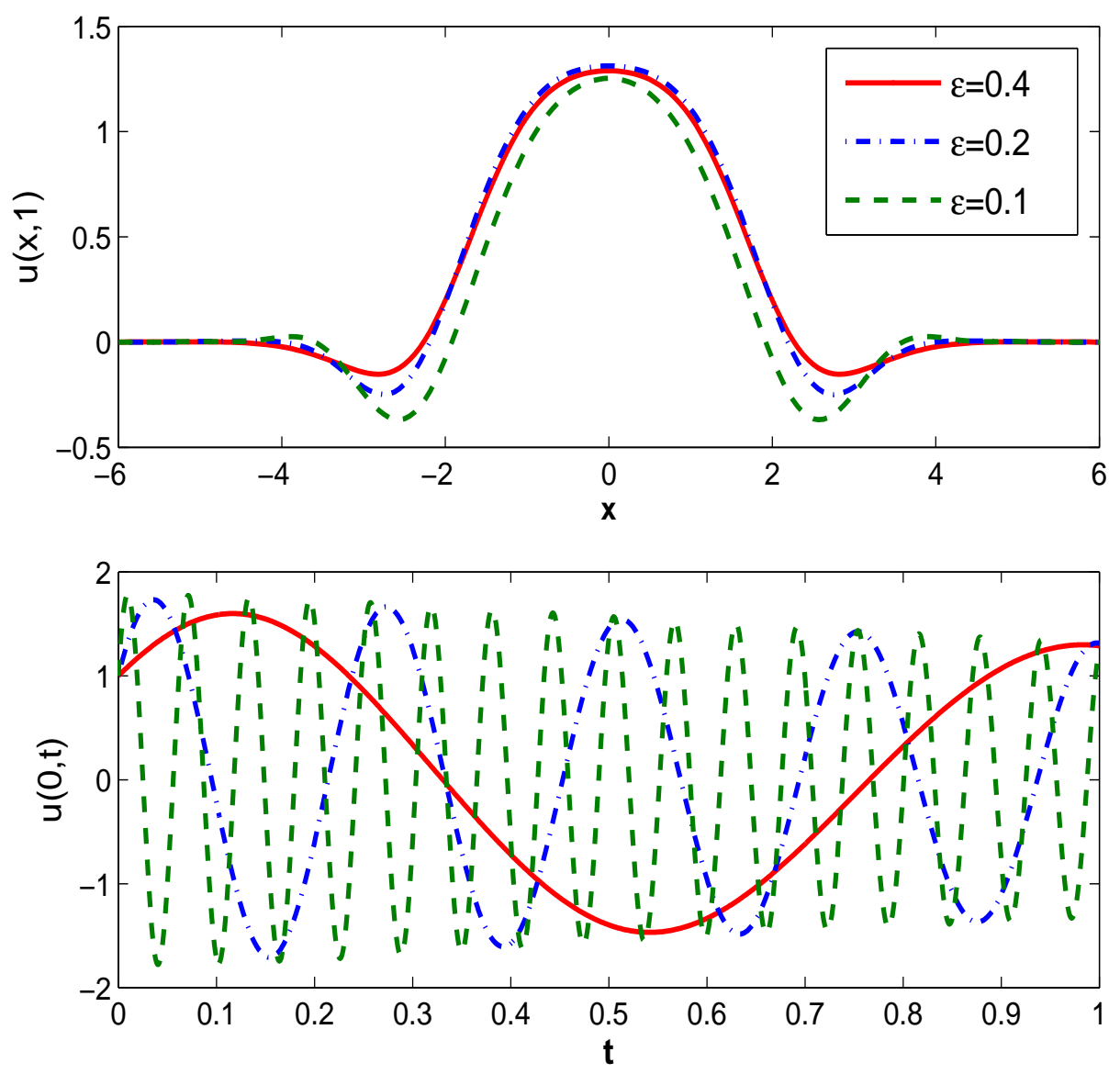

Fig. 1.1: The solution of (1.1) with $d=1, f(u)=|u|^{2} u, \phi_{1}(x)=e^{-x^{2} / 2}$ and $\phi_{2}(x)=$ $\frac{3}{2} \phi_{1}(x)$ for different $\varepsilon$.

proposed and analyzed two multiscale time integrators (MTIs) based on multiscale decompositions of the solution [6]. The two MTIs converge uniformly for $\varepsilon \in(0,1]$ and have some advantages compared to the FDTD and EWI as well as asymptotic preserving methods in integrating highly oscillatory second-order ODEs for $\varepsilon \in(0,1][6]$, especially when $\varepsilon$ is not too big and too small, i.e. in the intermediate regime. The aim of this paper is to design and analyze a multiscale time integrator Fourier pseudospectral (MTI-FP) method for the problem (1.1) based on a multiscale decomposition of the solution of (1.1) $28,30,32$ and the MTIs to the highly oscillatory second-order ODEs in [6]. The proposed MTI-FP method to (1.1) is explicit, efficient and accurate in practical computation, and converges in time uniformly at linear convergence rate for all $\varepsilon \in(0,1]$ and optimally at quadratic convergence rate in the regimes $\varepsilon=O(1)$ or $0<\varepsilon \leq \tau$. Thus our method is different with those numerical methods in [5,8,15].

The paper is organized as follows. In section 2 we introduce a multiscale decomposition for the KG equation (1.1) based on frequency. A MTI-FP method is proposed in section 3, and its rigorous error bounds are established in section 4 . 
Numerical results are reported in section 5. Finally, some conclusions are drawn in section 6] Throughout this paper, we adopt the standard Sobolev spaces [1] and use the notation $A \lesssim B$ to represent that there exists a generic constant $C>0$, which is independent of time step $\tau$ (or $n$ ), mesh size $h$ and $\varepsilon$, such that $|A| \leq C B$.

2. A multiscale decomposition. Let $\tau=\Delta t>0$ be the step size, and denote time steps by $t_{n}=n \tau$ for $n=0,1, \ldots$. In this section, we present a multiscale decomposition for the solution of (1.1) on the time interval $\left[t_{n}, t_{n+1}\right]$ with given initial data at $t=t_{n}$ as

$$
u\left(\mathbf{x}, t_{n}\right)=\phi_{1}^{n}(\mathbf{x})=O(1), \quad \partial_{t} u\left(\mathbf{x}, t_{n}\right)=\frac{1}{\varepsilon^{2}} \phi_{2}^{n}(\mathbf{x})=O\left(\frac{1}{\varepsilon^{2}}\right) .
$$

Similarly to the analytical study of the nonrelativistic limit of the nonlinear KG equation in 29, 30, we take an ansatz to the solution $u(\mathbf{x}, t):=u\left(\mathbf{x}, t_{n}+s\right)$ of (1.1) on the time interval $\left[t_{n}, t_{n+1}\right]$ with (2.1) as [6]

$$
u\left(\mathbf{x}, t_{n}+s\right)=e^{i s / \varepsilon^{2}} z_{+}^{n}(\mathbf{x}, s)+e^{-i s / \varepsilon^{2} \overline{z_{-}^{n}}}(\mathbf{x}, s)+r^{n}(\mathbf{x}, s), \quad \mathbf{x} \in \mathbb{R}^{d}, \quad 0 \leq s \leq \tau .
$$

Here and after, $\bar{z}$ denotes the complex conjugate of a complex-valued function $z$. Differentiating (2.2) with respect to $s$, we have

$$
\begin{aligned}
\partial_{s} u\left(\mathbf{x}, t_{n}+s\right)= & e^{i s / \varepsilon^{2}}\left[\partial_{s} z_{+}^{n}(\mathbf{x}, s)+\frac{i}{\varepsilon^{2}} z_{+}^{n}(\mathbf{x}, s)\right]+e^{-i s / \varepsilon^{2}}\left[\partial_{s} \overline{z_{-}^{n}}(\mathbf{x}, s)-\frac{i}{\varepsilon^{2}} \overline{z_{-}^{n}}(\mathbf{x}, s)\right] \\
& +\partial_{s} r^{n}(\mathbf{x}, s), \quad \mathbf{x} \in \mathbb{R}^{d}, \quad 0 \leq s \leq \tau .
\end{aligned}
$$

Plugging (2.2) into (1.1), we get for $\mathbf{x} \in \mathbb{R}^{d}$ and $0 \leq s \leq \tau$

$$
\begin{aligned}
& e^{i s / \varepsilon^{2}}\left[\varepsilon^{2} \partial_{s s} z_{+}^{n}(\mathbf{x}, s)+2 i \partial_{s} z_{+}^{n}(\mathbf{x}, s)-\Delta z_{+}^{n}(\mathbf{x}, s)\right]+\varepsilon^{2} \partial_{s s} r^{n}(\mathbf{x}, s)+\Delta r^{n}(\mathbf{x}, s) \\
& +e^{-i s / \varepsilon^{2}}\left[\varepsilon^{2} \partial_{s s} \overline{z_{-}^{n}}(\mathbf{x}, s)-2 i \partial_{s} \overline{z_{-}^{n}}(\mathbf{x}, s)-\Delta \overline{z_{-}^{n}}(\mathbf{x}, s)\right]+\frac{r^{n}(\mathbf{x}, s)}{\varepsilon^{2}}+f\left(u\left(\mathbf{x}, t_{n}+s\right)\right)=0 .
\end{aligned}
$$

Multiplying the above equation by $e^{-i s / \varepsilon^{2}}$ and $e^{i s / \varepsilon^{2}}$, respectively, we can decompose it into a coupled system for two $\varepsilon^{2}$-frequency waves with the unknowns $z_{ \pm}^{n}(\mathbf{x}, s):=z_{ \pm}^{n}$ and the rest frequency waves with the unknown $r^{n}(\mathbf{x}, s):=r^{n}$ as

$$
\left\{\begin{array}{l}
\varepsilon^{2} \partial_{s s} z_{ \pm}^{n}+2 i \partial_{s} z_{ \pm}^{n}-\Delta z_{ \pm}^{n}+f_{ \pm}\left(z_{+}^{n}, z_{-}^{n}\right)=0, \\
\varepsilon^{2} \partial_{s s} r^{n}-\Delta r^{n}+\frac{1}{\varepsilon^{2}} r^{n}+f_{r}\left(z_{+}^{n}, z_{-}^{n}, r^{n} ; s\right)=0,
\end{array} \quad \mathbf{x} \in \mathbb{R}^{d}, \quad 0 \leq s \leq \tau,\right.
$$

where

$$
\begin{aligned}
& f_{ \pm}\left(z_{+}, z_{-}\right)=\frac{1}{2 \pi} \int_{0}^{2 \pi} f\left(z_{ \pm}+e^{i \theta} \overline{z_{\mp}}\right) d \theta, \quad z_{ \pm}, r \in \mathbb{C}, \quad 0 \leq s \leq \tau \\
& f_{r}\left(z_{+}, z_{-}, r ; s\right)=f\left(e^{i s / \varepsilon^{2}} z_{+}+e^{-i s / \varepsilon^{2}} \overline{z_{-}}+r\right)-f_{+}\left(z_{+}, z_{-}\right) e^{i s / \varepsilon^{2}}-\overline{f_{-}}\left(z_{+}, z_{-}\right) e^{-i s / \varepsilon^{2}} .
\end{aligned}
$$

In order to find proper initial conditions for the above system (2.4), setting $s=0$ in (2.2) and (2.3), noticing (2.1), we obtain

$$
\left\{\begin{array}{l}
z_{+}^{n}(\mathbf{x}, 0)+\overline{z_{-}^{n}}(\mathbf{x}, 0)+r^{n}(\mathbf{x}, 0)=\phi_{1}^{n}(\mathbf{x}), \quad \mathbf{x} \in \mathbb{R}^{d}, \\
\frac{i}{\varepsilon^{2}}\left[z_{+}^{n}(\mathbf{x}, 0)-\overline{z_{-}^{n}}(\mathbf{x}, 0)\right]+\partial_{s} z_{+}^{n}(\mathbf{x}, 0)+\partial_{s} \overline{z_{-}^{n}}(\mathbf{x}, 0)+\partial_{s} r^{n}(\mathbf{x}, 0)=\frac{\phi_{2}^{n}(\mathbf{x})}{\varepsilon^{2}} .
\end{array}\right.
$$


Now we decompose the above initial data so as to: (i) equate $O\left(\frac{1}{\varepsilon^{2}}\right)$ and $O(1)$ terms in the second equation of (2.5), respectively, and (ii) be well-prepared for the first two equations in (2.4) when $0<\varepsilon \ll 1$, i.e. $\partial_{s} z_{+}^{n}(\mathbf{x}, 0)$ and $\partial_{s} z_{-}^{n}(\mathbf{x}, 0)$ are determined from the first two equations in (2.4), respectively, by setting $\varepsilon=0$ and $s=0$ [3, 4 :

$$
\left\{\begin{array}{l}
z_{+}^{n}(\mathbf{x}, 0)+\overline{z_{-}^{n}}(\mathbf{x}, 0)=\phi_{1}^{n}(\mathbf{x}), \quad i\left[z_{+}^{n}(\mathbf{x}, 0)-\overline{z_{-}^{n}}(\mathbf{x}, 0)\right]=\phi_{2}^{n}(\mathbf{x}), \\
2 i \partial_{s} z_{ \pm}^{n}(\mathbf{x}, 0)-\Delta z_{ \pm}^{n}(\mathbf{x}, 0)+f_{ \pm}\left(z_{+}^{n}(\mathbf{x}, 0), z_{-}^{n}(\mathbf{x}, 0)\right)=0, \\
r^{n}(\mathbf{x}, 0)=0, \quad \partial_{s} r^{n}(\mathbf{x}, 0)+\partial_{s} z_{+}^{n}(\mathbf{x}, 0)+\partial_{s} \overline{z_{-}^{n}}(\mathbf{x}, 0)=0
\end{array} \quad \mathbf{x} \in \mathbb{R}^{d}\right.
$$

Solving (2.6), we get the initial data for (2.4) as

$$
\left\{\begin{array}{l}
z_{+}^{n}(\mathbf{x}, 0)=\frac{1}{2}\left[\phi_{1}^{n}(\mathbf{x})-i \phi_{2}^{n}(\mathbf{x})\right], \quad z_{-}^{n}(\mathbf{x}, 0)=\frac{1}{2}\left[\overline{\phi_{1}^{n}}(\mathbf{x})-i \overline{\phi_{2}^{n}}(\mathbf{x})\right] \\
\partial_{s} z_{ \pm}^{n}(\mathbf{x}, 0)=\frac{i}{2}\left[-\Delta z_{ \pm}^{n}(\mathbf{x}, 0)+f_{ \pm}\left(z_{+}^{n}(\mathbf{x}, 0), z_{-}^{n}(\mathbf{x}, 0)\right)\right], \quad \mathbf{x} \in \mathbb{R}^{d}, \\
r^{n}(\mathbf{x}, 0)=0, \quad \partial_{s} r^{n}(\mathbf{x}, 0)=-\partial_{s} z_{+}^{n}(\mathbf{x}, 0)-\partial_{s} \overline{z_{-}^{n}}(\mathbf{x}, 0) .
\end{array}\right.
$$

The above decomposition (2.2) can be called as multiscale decomposition by frequency (MDF). In fact, it can also be regarded as to decompose slow waves at $\varepsilon^{2}$-wavelength and fast waves at other wavelengths, thus it can also be called as fast-slow frequency decomposition (FSFD). On the other hand, the amplitude of $z_{ \pm}^{n}$ is usually at $O(1)$ and the amplitude of $r^{n}$ is at $O\left(\varepsilon^{2}\right)=o(1)$ when $\varepsilon$ is small, thus it can also be regarded as large-small amplitude decomposition (LSAD). Specifically, for the pure power nonlinearity, i.e. $f$ satisfies (1.3), explicit formulas for $f_{ \pm}$and $f_{r}$ have been given in 6 .

After solving the decomposed system (2.4) with the initial data (2.7), we get $z_{ \pm}^{n}(\mathbf{x}, \tau), \partial_{s} z_{ \pm}^{n}(\mathbf{x}, \tau), r^{n}(\mathbf{x}, \tau)$ and $\partial_{s} r^{n}(\mathbf{x}, \tau)$. Then we can reconstruct the solution to (1.1) at $t=t_{n+1}$ by setting $s=\tau$ in (2.2) and (2.3), i.e.,

$$
\left\{\begin{array}{l}
u\left(\mathbf{x}, t_{n+1}\right)=e^{i \tau / \varepsilon^{2}} z_{+}^{n}(\mathbf{x}, \tau)+e^{-i \tau / \varepsilon^{2}} z_{-}^{n}(\mathbf{x}, \tau)+r^{n}(\mathbf{x}, \tau):=\phi_{1}^{n+1}(\mathbf{x}), \\
\partial_{t} u\left(\mathbf{x}, t_{n+1}\right)=\frac{1}{\varepsilon^{2}} \phi_{2}^{n+1}(\mathbf{x}), \quad \mathbf{x} \in \mathbb{R}^{d}
\end{array}\right.
$$

with

$\phi_{2}^{n+1}(\mathbf{x})=e^{i \tau / \varepsilon^{2}}\left[\varepsilon^{2} \partial_{s} z_{+}^{n}(\mathbf{x}, \tau)+i z_{+}^{n}(\mathbf{x}, \tau)\right]+e^{-i \tau / \varepsilon^{2}}\left[\varepsilon^{2} \partial_{s} \overline{z_{-}^{n}}(\mathbf{x}, \tau)-i \overline{z_{-}^{n}}(\mathbf{x}, \tau)\right]+\varepsilon^{2} \partial_{s} r^{n}(\mathbf{x}, \tau)$.

3. A MTI-FP method. In this section, based on the MDF (2.4), we propose a new numerical method for solving the KG equation (1.1) with the pure power nonlinearity (1.3), which is uniformly accurate for $\varepsilon \in(0,1]$. For the simplicity of notations, we present the numerical method in one space dimension (1D) with a cubic nonlinearity, i.e. $d=1$ in (1.1) and $f(u)=\lambda|u|^{2} u$ with $\lambda \in \mathbb{R}$ a given constant in (1.3). In this case, we have

$$
\left\{\begin{array}{l}
f_{ \pm}\left(z_{+}, z_{-}\right)=\lambda\left(\left|z_{ \pm}\right|^{2}+2\left|z_{\mp}\right|^{2}\right) z_{ \pm}, \quad z_{ \pm}, r \in \mathbb{C}, \quad 0 \leq s \leq \tau, \\
f_{r}\left(z_{+}, z_{-}, r ; s\right)=e^{3 i s / \varepsilon^{2}} g_{+}\left(z_{+}, z_{-}\right)+e^{-3 i s / \varepsilon^{2}} \overline{g_{-}}\left(z_{+}, z_{-}\right)+w\left(z_{+}, z_{-}, r ; s\right),
\end{array}\right.
$$

with

$$
\left\{\begin{array}{l}
g_{ \pm}\left(z_{+}, z_{-}\right)=\lambda z_{ \pm}^{2} z_{\mp}, \quad z_{ \pm}, r \in \mathbb{C}, \quad 0 \leq s \leq \tau \\
w\left(z_{+}, z_{-}, r ; s\right)=f\left(e^{i s / \varepsilon^{2}} z_{+}+e^{-i s / \varepsilon^{2}} \overline{z_{-}}+r\right)-f\left(e^{i s / \varepsilon^{2}} z_{+}+e^{-i s / \varepsilon^{2}} \overline{z_{-}}\right) .
\end{array}\right.
$$


Generalizations to higher dimensions and general pure power nonlinearity are straightforward and all the results presented in this paper are still valid with minor modifications. Due to fast decay of the solution to the KG equation (1.1) at far field, similar to those in the literature for numerical computations [5, 7, 10, 14, 15, 26, 39, the whole space problem (1.1) in $1 \mathrm{D}$ is usually truncated onto a finite interval $\Omega=(a, b)$ with periodic boundary conditions ( $a$ and $b$ are usually chosen sufficient large such that the truncation error is negligible):

$$
\left\{\begin{array}{l}
\varepsilon^{2} \partial_{t t} u(x, t)-\partial_{x x} u(x, t)+\frac{u(x, t)}{\varepsilon^{2}}+f(u(x, t))=0, \quad x \in \Omega=(a, b), t>0 \\
u(a, t)=u(b, t), \quad \partial_{x} u(a, t)=\partial_{x} u(b, t), \quad t \geq 0 \\
u(x, 0)=\phi_{1}(x), \quad \partial_{t} u(x, 0)=\frac{1}{\varepsilon^{2}} \phi_{2}(x), \quad x \in \bar{\Omega}=[a, b] .
\end{array}\right.
$$

Consequently, for $n \geq 0$, the decomposed system MDF (2.4) in 1D collapses to

$$
\left\{\begin{array}{l}
\varepsilon^{2} \partial_{s s} z_{ \pm}^{n}+2 i \partial_{s} z_{ \pm}^{n}-\partial_{x x} z_{ \pm}^{n}+f_{ \pm}\left(z_{+}^{n}, z_{-}^{n}\right)=0, \\
\varepsilon^{2} \partial_{s s} r^{n}-\partial_{x x} r^{n}+\frac{1}{\varepsilon^{2}} r^{n}+f_{r}\left(z_{+}^{n}, z_{-}^{n}, r^{n} ; s\right)=0, \quad a<x<b, 0<s \leq \tau .
\end{array}\right.
$$

The initial and boundary conditions for the above system are

$$
\left\{\begin{array}{l}
z_{ \pm}^{n}(a, s)=z_{ \pm}^{n}(b, s), \quad \partial_{x} z_{ \pm}^{n}(a, s)=\partial_{x} z_{ \pm}^{n}(b, s), \\
r^{n}(a, s)=r^{n}(b, s), \quad \partial_{x} r^{n}(a, s)=\partial_{x} r^{n}(b, s), \quad 0 \leq s \leq \tau ; \\
z_{+}^{n}(x, 0)=\frac{1}{2}\left[\phi_{1}^{n}(x)-i \phi_{2}^{n}(x)\right], \quad z_{-}^{n}(x, 0)=\frac{1}{2}\left[\overline{\phi_{1}^{n}}(x)-i \overline{\phi_{2}^{n}}(x)\right], \\
\partial_{s} z_{ \pm}^{n}(x, 0)=\frac{i}{2}\left[-\partial_{x x} z_{ \pm}^{n}(x, 0)+f_{ \pm}\left(z_{+}^{n}(x, 0), z_{-}^{n}(x, 0)\right)\right], \quad a \leq x \leq b, \\
r^{n}(x, 0)=0, \quad \partial_{s} r^{n}(x, 0)=-\partial_{s} z_{+}^{n}(x, 0)-\partial_{s} \overline{z_{-}^{n}}(x, 0) .
\end{array}\right.
$$

In order to discretize (3.4) with (3.5), we first apply the Fourier spectral method in space and then use the exponential wave integrator (EWI) for time integration [6]. Choose the mesh size $h:=\Delta x=(b-a) / N$ with $N$ a positive integer and denote grid points as $x_{j}:=a+j h$ for $j=0,1, \ldots, N$. Define

$$
\begin{aligned}
& X_{N}:=\operatorname{span}\left\{\phi_{l}(x)=e^{i \mu_{l}(x-a)} \mid l=-\frac{N}{2}, \ldots, \frac{N}{2}-1\right\} \quad \text { with } \mu_{l}=\frac{2 \pi l}{b-a}, \\
& Y_{N}:=\left\{\mathbf{v}=\left(v_{0}, v_{1}, \ldots, v_{N}\right) \in \mathbb{C}^{N+1} \mid v_{0}=v_{N}\right\} \quad \text { with }\|\mathbf{v}\|_{l^{2}}=h \sum_{j=0}^{N-1}\left|v_{j}\right|^{2} .
\end{aligned}
$$

For a periodic function $v(x)$ on $\bar{\Omega}$ and a vector $\mathbf{v} \in Y_{N}$, let $P_{N}: L^{2}(\Omega) \rightarrow X_{N}$ be the standard $L^{2}$-projection operator, and $I_{N}: C(\Omega) \rightarrow X_{N}$ or $Y_{N} \rightarrow X_{N}$ be the trigonometric interpolation operator [38, i.e.

$$
\left(P_{N} v\right)(x)=\sum_{l=-N / 2}^{N / 2-1} \widehat{v}_{l} e^{i \mu_{l}(x-a)}, \quad\left(I_{N} \mathbf{v}\right)(x)=\sum_{l=-N / 2}^{N / 2-1} \widetilde{v}_{l} e^{i \mu_{l}(x-a)}, \quad a \leq x \leq b,
$$

where $\widehat{v}_{l}$ and $\widetilde{v}_{l}$ are the Fourier and discrete Fourier transform coefficients of the periodic function $v(x)$ and vector $\mathbf{v}$, respectively, defined as

$$
\widehat{v}_{l}=\frac{1}{b-a} \int_{a}^{b} v(x) e^{-i \mu_{l}(x-a)} d x, \quad \widetilde{v}_{l}=\frac{1}{N} \sum_{j=0}^{N-1} v_{j} e^{-i \mu_{l}\left(x_{j}-a\right)} .
$$


Then a Fourier spectral method for discretizing (3.4) reads:

Find $z_{ \pm, N}^{n}:=z_{ \pm, N}^{n}(x, s), r_{N}^{n}:=r_{N}^{n}(x, s) \in X_{N}$ for $0 \leq s \leq \tau$, i.e.

$$
z_{ \pm, N}^{n}(x, s)=\sum_{l=-N / 2}^{N / 2-1} \widehat{\left(z_{ \pm}^{n}\right)_{l}}(s) e^{i \mu_{l}(x-a)}, \quad r_{N}^{n}(x, s)=\sum_{l=-N / 2}^{N / 2-1} \widehat{\left(r^{n}\right)_{l}}(s) e^{i \mu_{l}(x-a)}
$$

such that for $0<s<\tau$

$$
\left\{\begin{array}{l}
\varepsilon^{2} \partial_{s s} z_{ \pm, N}^{n}+2 i \partial_{s} z_{ \pm, N}^{n}-\partial_{x x} z_{ \pm, N}^{n}+P_{N} f_{ \pm}\left(z_{+, N}^{n}, z_{-, N}^{n}\right)=0, \quad a<x<b \\
\varepsilon^{2} \partial_{s s} r_{N}^{n}-\partial_{x x} r_{N}^{n}+\frac{1}{\varepsilon^{2}} r_{N}^{n}+P_{N} f_{r}\left(z_{+, N}^{n}, z_{-, N}^{n}, r_{N}^{n} ; s\right)=0
\end{array}\right.
$$

Substituting (3.8) into (3.9) and noticing the orthogonality of $\phi_{l}(x)$, we get

$$
\left\{\begin{array}{l}
\varepsilon^{2} \widehat{\left(z_{ \pm}^{n}\right)_{l}^{\prime \prime}}(s)+2 i \widehat{\left(z_{ \pm}^{n}\right)_{l}^{\prime}}(s)+\mu_{l}^{2} \widehat{\left.z_{ \pm}^{n}\right)_{l}}(s)+\widehat{\left(f_{ \pm}^{n}\right)_{l}}(s)=0, \quad 0<s \leq \tau, \\
\varepsilon^{2}{\widehat{\left(r^{n}\right)_{l}^{\prime \prime}}}_{l}^{\prime \prime}(s)+\left(\mu_{l}^{2}+\frac{1}{\varepsilon^{2}}\right) \widehat{\left(r^{n}\right)_{l}}(s)+\widehat{\left(f_{r}^{n}\right)_{l}}(s)=0, \quad-\frac{N}{2} \leq l \leq \frac{N}{2}-1,
\end{array}\right.
$$

where $\widehat{\left(f_{ \pm}^{n}\right)_{l}}(s)$ and $\widehat{\left(f_{r}^{n}\right)_{l}}(s)$ are the Fourier coefficients of $f_{ \pm}^{n}(x, s):=f_{ \pm}\left(z_{+, N}^{n}(x, s)\right.$, $\left.z_{-, N}^{n}(x, s)\right)$ and $f_{r}^{n}(x, s):=f_{r}\left(z_{+, N}^{n}(x, s), z_{-, N}^{n}(x, s), r_{N}^{n}(x, s) ; s\right)$, respectively. In order to apply the EWIs for integrating (3.10) in time, for each fixed $-N / 2 \leq l \leq$ $N / 2-1$, we re-write (3.10) by using the variation-of-constant formulas

$$
\left\{\begin{array}{l}
\widehat{\left(z_{ \pm}^{n}\right)_{l}}(s)=a_{l}(s) \widehat{\left(z_{ \pm}^{n}\right)}(0)+\varepsilon^{2} b_{l}(s) \widehat{\left(z_{ \pm}^{n}\right)_{l}^{\prime}}(0)-\int_{0}^{s} b_{l}(s-\theta) \widehat{\left(f_{ \pm}^{n}\right)_{l}}(\theta) d \theta, \\
\widehat{\left(r^{n}\right)_{l}}(s)=\frac{\sin \left(\omega_{l} s\right)}{\omega_{l}}{\widehat{\left(r^{n}\right.}}_{l}^{\prime}(0)-\int_{0}^{s} \frac{\sin \left(\omega_{l}(s-\theta)\right)}{\varepsilon^{2} \omega_{l}}\left(\widehat{\left.f_{r}^{n}\right)_{l}}(\theta) d \theta, \quad 0 \leq s \leq \tau\right.
\end{array}\right.
$$

where $\omega_{l}=\frac{1}{\varepsilon^{2}} \sqrt{1+\mu_{l}^{2} \varepsilon^{2}}$ and

$$
\left\{\begin{array}{l}
a_{l}(s):=\frac{\lambda_{l}^{+} e^{i s \lambda_{l}^{-}}-\lambda_{l}^{-} e^{i s \lambda_{l}^{+}}}{\lambda_{l}^{+}-\lambda_{l}^{-}}, \quad b_{l}(s):=i \frac{e^{i s \lambda_{l}^{+}}-e^{i s \lambda_{l}^{-}}}{\varepsilon^{2}\left(\lambda_{l}^{-}-\lambda_{l}^{+}\right)}, \quad 0 \leq s \leq \tau, \\
\lambda_{l}^{+}=-\frac{1+\sqrt{1+\mu_{l}^{2} \varepsilon^{2}}}{\varepsilon^{2}}=O\left(\frac{1}{\varepsilon^{2}}\right), \quad \lambda_{l}^{-}=-\frac{1-\sqrt{1+\mu_{l}^{2} \varepsilon^{2}}}{\varepsilon^{2}}=O(1) .
\end{array}\right.
$$

Differentiating (3.11) with respect to $s$, we obtain

$$
\left\{\begin{array}{l}
\widehat{\left(z_{ \pm}^{n}\right)_{l}^{\prime}}(s)=a_{l}^{\prime}(s) \widehat{\left(z_{ \pm}^{n}\right)_{l}}(0)+\varepsilon^{2} b_{l}^{\prime}(s) \widehat{\left(z_{ \pm}^{n}\right)_{l}^{\prime}}(0)-\int_{0}^{s} b_{l}^{\prime}(s-\theta) \widehat{\left(f_{ \pm}^{n}\right)_{l}}(\theta) d \theta, \\
\widehat{\left(r^{n}\right)_{l}^{\prime}}(s)=\cos \left(\omega_{l} s\right){\widehat{\left(r^{n}\right)_{l}}}_{l}^{\prime}(0)-\int_{0}^{s} \frac{\cos \left(\omega_{l}(s-\theta)\right)}{\varepsilon^{2}}\left(\widehat{\left.f_{r}^{n}\right)_{l}}(\theta) d \theta, \quad 0 \leq s \leq \tau,\right.
\end{array}\right.
$$

where

$$
a_{l}^{\prime}(s)=i \lambda_{l}^{+} \lambda_{l}^{-} \frac{e^{i s \lambda_{l}^{-}}-e^{i s \lambda_{l}^{+}}}{\lambda_{l}^{+}-\lambda_{l}^{-}}, \quad b_{l}^{\prime}(s)=\frac{\lambda_{l}^{+} e^{i s \lambda_{l}^{+}}-\lambda_{l}^{-} e^{i s \lambda_{l}^{-}}}{\varepsilon^{2}\left(\lambda_{l}^{+}-\lambda_{l}^{-}\right)}, \quad 0 \leq s \leq \tau .
$$

Taking $s=\tau$ in (3.11) and (3.13), noticing (3.1) and (3.2), and approximating the integrals either by the Gautschi's type quadrature [6,17,24] or by the standard trape- 
zoidal rule [6, 17, 24, we get

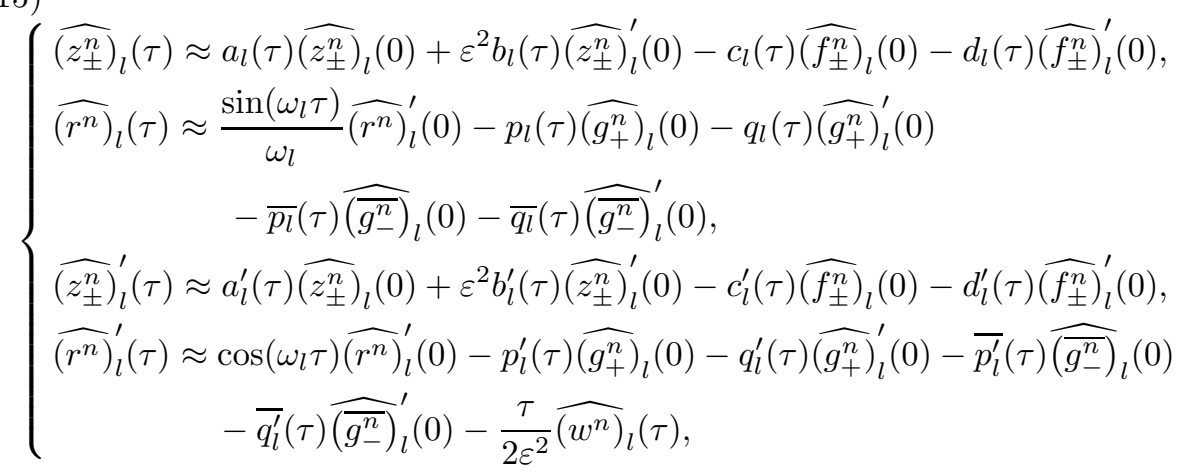

where $\widehat{\left(g_{ \pm}^{n}\right)_{l}}(s), \widehat{\left(w^{n}\right)_{l}}(s), \widehat{\left(f_{ \pm}^{n}\right)_{l}^{\prime}}(s)=\widehat{\left(\partial_{s} f_{ \pm}^{n}\right)_{l}}(s)$ and ${\widehat{\left(g_{ \pm}^{n}\right)_{l}}}_{l}^{\prime}(s)=\widehat{\left(\partial_{s} g_{ \pm}^{n}\right)_{l}}(s)$ are the Fourier coefficients of $g_{ \pm}^{n}=g_{ \pm}\left(z_{+, N}^{n}, z_{-, N}^{n}\right), w^{n}=w\left(z_{+, N}^{n}, z_{-, N}^{n}, r_{N}^{n} ; s\right), \partial_{s} f_{ \pm}^{n}=$ $2 \lambda z_{ \pm}^{n} \operatorname{Re}\left[\overline{z_{ \pm}^{n}} \partial_{s} z_{ \pm}^{n}+2 \overline{z_{\mp}^{n}} \partial_{s} z_{\mp}^{n}\right]+\lambda \partial_{s} z_{ \pm}^{n}\left[\left|z_{ \pm}^{n}\right|^{2}+2\left|z_{\mp}^{n}\right|^{2}\right]=: \dot{f}_{ \pm}\left(z_{+}^{n}, z_{-}^{n} ; \partial_{s} z_{+}^{n}, \partial_{s} z_{-}^{n}\right)$ and $\partial_{s} g_{ \pm}^{n}=2 \lambda z_{ \pm}^{n} z_{\mp}^{n} \partial_{s} z_{ \pm}^{n}+\lambda\left(z_{ \pm}^{n}\right)^{2} \partial_{s} z_{\mp}^{n}=: \dot{g}_{ \pm}\left(z_{+}^{n}, z_{-}^{n} ; \partial_{s} z_{+}^{n}, \partial_{s} z_{-}^{n}\right)$, respectively, and (their detailed explicit formulas are shown in [6, appendix])

$$
\begin{cases}c_{l}(\tau)=\int_{0}^{\tau} b_{l}(\tau-\theta) d \theta, & p_{l}(\tau)=\int_{0}^{\tau} \frac{\sin \left(\omega_{l}(\tau-\theta)\right)}{\varepsilon^{2} \omega_{l}} e^{3 i \theta / \varepsilon^{2}} d \theta, \\ d_{l}(\tau)=\int_{0}^{\tau} b_{l}(\tau-\theta) \theta d \theta, & q_{l}(\tau)=\int_{0}^{\tau} \frac{\sin \left(\omega_{l}(\tau-\theta)\right)}{\varepsilon^{2} \omega_{l}} e^{3 i \theta / \varepsilon^{2}} \theta d \theta, \\ c_{l}^{\prime}(\tau)=\int_{0}^{\tau} b_{l}^{\prime}(\tau-\theta) d \theta, & p_{l}^{\prime}(\tau)=\int_{0}^{\tau} \frac{\cos \left(\omega_{l}(\tau-\theta)\right)}{\varepsilon^{2}} e^{3 i \theta / \varepsilon^{2}} d \theta, \\ d_{l}^{\prime}(\tau)=\int_{0}^{\tau} b_{l}^{\prime}(\tau-\theta) \theta d \theta, & q_{l}^{\prime}(\tau)=\int_{0}^{\tau} \frac{\cos \left(\omega_{l}(\tau-\theta)\right)}{\varepsilon^{2}} e^{3 i \theta / \varepsilon^{2}} \theta d \theta .\end{cases}
$$

Inserting (3.15) into (3.8) and its time derivative with setting $s=\tau$, and noticing (2.8), we immediately obtain a MTI-FP discretization for the problem (3.3).

In practice, the integrals for computing the Fourier transform coefficients in (3.7), (3.11) and (3.13) are usually approximated by numerical quadratures [4,5, 38, Let $u_{j}^{n}$ and $\dot{u}_{j}^{n}$ be approximations of $u\left(x_{j}, t_{n}\right)$ and $\partial_{t} u\left(x_{j}, t_{n}\right)$, respectively; and $z_{ \pm, j}^{n+1}, \dot{z}_{ \pm, j}^{n+1}$, $r_{j}^{n+1}$ and $\dot{r}_{j}^{n+1}$ be approximations of $z_{ \pm}^{n}\left(x_{j}, \tau\right), \partial_{s} z_{ \pm}^{n}\left(x_{j}, \tau\right), r^{n}\left(x_{j}, \tau\right)$ and $\partial_{s} r^{n}\left(x_{j}, \tau\right)$, respectively, for $j=0, \ldots, N$. Choosing $u_{j}^{0}=\phi_{1}\left(x_{j}\right)$ and $\dot{u}_{j}^{0}=\phi_{2}\left(x_{j}\right) / \varepsilon^{2}$ for $0 \leq$ $j \leq N$ and noticing (2.8), (3.8) with $s=\tau$, (3.15), (3.5) and (2.1), then a MTI-FP discretization for the problem (3.3) reads for $n \geq 0$

$$
\left\{\begin{array}{l}
u_{j}^{n+1}=e^{i \tau / \varepsilon^{2}} z_{+, j}^{n+1}+e^{-i \tau / \varepsilon^{2}} \overline{z_{-, j}^{n+1}}+r_{j}^{n+1}, \quad j=0,1, \ldots, N, \\
\dot{u}_{j}^{n+1}=e^{i \tau / \varepsilon^{2}}\left(\dot{z}_{+, j}^{n+1}+\frac{i}{\varepsilon^{2}} z_{+, j}^{n+1}\right)+e^{-i \tau / \varepsilon^{2}}\left(\overline{\dot{z}_{-, j}^{n+1}}-\frac{i}{\varepsilon^{2}} \overline{z_{-, j}^{n+1}}\right)+\dot{r}_{j}^{n+1},
\end{array}\right.
$$

where

$$
\begin{cases}z_{ \pm, j}^{n+1}=\sum_{l=-N / 2}^{N / 2-1} \widetilde{\left(z_{ \pm}^{n+1}\right)_{l}} e^{i \mu_{l}\left(x_{j}-a\right)}, & r_{j}^{n+1}=\sum_{l=-N / 2}^{N / 2-1} \widetilde{\left(r^{n+1}\right)_{l}} e^{i \mu_{l}\left(x_{j}-a\right)}, \\ \dot{z}_{ \pm, j}^{n+1}=\sum_{l=-N / 2}^{N / 2-1} \widetilde{\left(\dot{z}_{ \pm}^{n+1}\right)_{l}} e^{i \mu_{l}\left(x_{j}-a\right)}, & \dot{r}_{j}^{n+1}=\sum_{l=-N / 2}^{N / 2-1} \widetilde{\left(\dot{r}^{n+1}\right)_{l}} e^{i \mu_{l}\left(x_{j}-a\right)},\end{cases}
$$


with

(3.19)

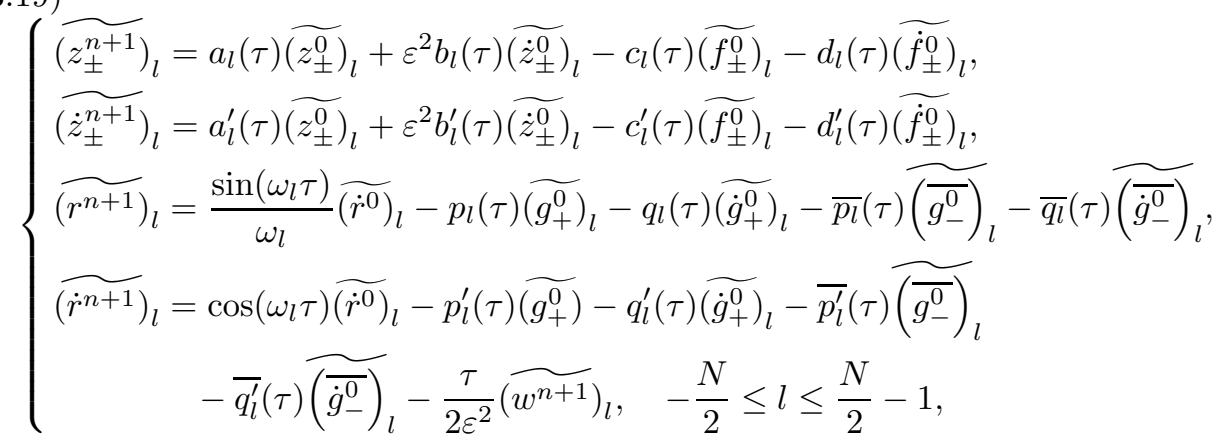

and

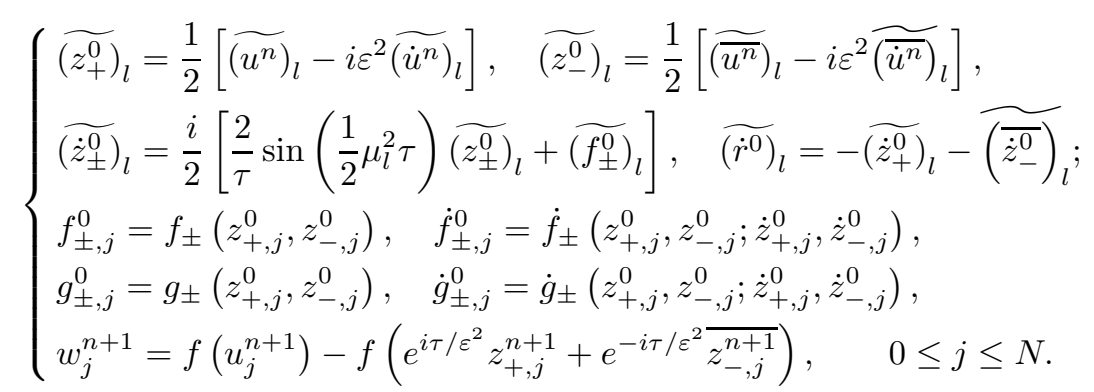

This MTI-FP method for the KG equation (3.3) (or (1.1)) is explicit, accurate, easy to implement and very efficient due to the fast Fourier transform (FFT), and its memory cost is $O(N)$ and the computational cost per time step is $O(N \log N)$.

REMARK 3.1. Instead of discretizing the initial velocity $\partial_{s} z_{ \pm}^{n}(x, 0)$ from (3.5) in Fourier space as $\widetilde{\left(z_{ \pm}^{n}\right)_{l}^{\prime}}(0)=\frac{i}{2}\left[\mu_{l}^{2}\left(\widetilde{\left.z_{ \pm}^{n}\right)_{l}}(0)+\widetilde{\left(f_{ \pm}^{n}\right)_{l}}(0)\right]\right.$ which will result a second order decreasing in the spatial accuracy, we change to the modified coefficients given in (3.20) as filters where the accuracy is now controlled by the time step $\tau$ (cf. (4.50) ). There are other possible choices of the filters.

REMARK 3.2. When the initial data $\phi_{1}(x)$ and $\phi_{2}(x)$ are real-valued functions and $f(u): \mathbb{R} \rightarrow \mathbb{R}$ in (1.1), then the solution $u(x, t)$ is real-valued. In this case, for $n \geq 0$, it is easy to see that $z_{+}^{n}(x, s)=z_{-}^{n}(x, s)$ for $x \in \Omega$ and $0 \leq s \leq \tau$ in the MDF 2.4). In the corresponding numerical scheme, we have $z_{+, j}^{n}=z_{-, j}^{n}$ for $j=0, \ldots, N$ in the MTI-FP (3.17). Thus the scheme can be simplified and the computational cost can be reduced.

4. Uniform convergence of MTI-FP. In this section, we establish an error bound for the MTI-FP (3.17) of the problem (3.3), which is uniformly for $\varepsilon \in(0,1]$. Let $0<T<T^{*}$ with $T^{*}$ the maximum existence time of the solution $u$ to the problem (3.3), motivated by the analytical results in [28 30], here we make the following assumption on the solution $u$ to the problem (3.3) - there exists an integer $m_{0} \geq 2$ such that

(A) $u \in C^{1}\left([0, T] ; H_{p}^{m_{0}+4}(\Omega)\right), \quad\|u\|_{L^{\infty}\left([0, T] ; H^{m_{0}+4}\right)}+\varepsilon^{2}\left\|\partial_{t} u\right\|_{L^{\infty}\left([0, T] ; H^{m_{0}+4}\right)} \lesssim 1$, where $H_{p}^{m}(\Omega)=\left\{\phi(x) \in H^{m}(\Omega) \mid \phi^{(k)}(a)=\phi^{(k)}(b), k=0,1, \ldots, m-1\right\} \subset H^{m}(\Omega)$. Denote

$$
C_{0}=\max _{0<\varepsilon \leq 1}\left\{\|u\|_{L^{\infty}\left([0, T] ; H^{m_{0}+4}\right)}, \varepsilon^{2}\left\|\partial_{t} u\right\|_{L^{\infty}\left([0, T] ; H^{m_{0}+4}\right)}\right\} .
$$


Let $\mathbf{u}^{n}=\left(u_{0}^{n}, u_{1}^{n}, \ldots, u_{N}^{n}\right) \in \mathbb{C}^{N+1}, \dot{\mathbf{u}}^{n}=\left(\dot{u}_{0}^{n}, \dot{u}_{1}^{n}, \ldots, \dot{u}_{N}^{n}\right) \in \mathbb{C}^{N+1}(n \geq 0)$ be the numerical solution obtained from the MTI-FP method (3.17), denote their interpolations as

$$
u_{I}^{n}(x):=\left(I_{N} \mathbf{u}^{n}\right)(x), \quad \dot{u}_{I}^{n}(x):=\left(I_{N} \dot{\mathbf{u}}^{n}\right)(x), \quad x \in \bar{\Omega},
$$

and define the error functions as

$$
e^{n}(x):=u\left(x, t_{n}\right)-u_{I}^{n}(x), \quad \dot{e}^{n}(x):=\partial_{t} u\left(x, t_{n}\right)-\dot{u}_{I}^{n}(x), \quad x \in \bar{\Omega}, 0 \leq n \leq \frac{T}{\tau},
$$

then we have the following error estimates for the MTI-FP method (3.17).

THEOREM 4.1 (Error bounds of MTI-FP). Under the assumption (A), there exist two constants $0<h_{0} \leq 1$ and $0<\tau_{0} \leq 1$ sufficiently small and independent of $\varepsilon$ such that, for any $0<\varepsilon \leq 1$, when $0<h \leq h_{0}$ and $0<\tau \leq \tau_{0}$, we have

$$
\begin{aligned}
& \left\|e^{n}\right\|_{H^{2}}+\varepsilon^{2}\left\|\dot{e}^{n}\right\|_{H^{2}} \lesssim h^{m_{0}}+\frac{\tau^{2}}{\varepsilon^{2}}, \quad\left\|e^{n}\right\|_{H^{2}}+\varepsilon^{2}\left\|\dot{e}^{n}\right\|_{H^{2}} \lesssim h^{m_{0}}+\tau^{2}+\varepsilon^{2} \\
& \left\|u_{I}^{n}\right\|_{H^{2}} \leq C_{0}+1, \quad\left\|\dot{u}_{I}^{n}\right\|_{H^{2}} \leq \frac{C_{0}+1}{\varepsilon^{2}}, \quad 0 \leq n \leq \frac{T}{\tau}
\end{aligned}
$$

Thus, by taking the minimum of the two error bounds in [4.4) for $\varepsilon \in(0,1]$, we obtain an error bound which is uniformly convergent for $\varepsilon \in(0,1]$

$$
\left\|e^{n}\right\|_{H^{2}}+\varepsilon^{2}\left\|\dot{e}^{n}\right\|_{H^{2}} \lesssim h^{m_{0}}+\tau^{2}+\min _{0<\varepsilon \leq 1}\left\{\frac{\tau^{2}}{\varepsilon^{2}}, \varepsilon^{2}\right\} \lesssim h^{m_{0}}+\tau, \quad 0 \leq n \leq \frac{T}{\tau} .
$$

In order to prove the above theorem, for $0 \leq n \leq \frac{T}{\tau}$, we introduce

$$
e_{N}^{n}(x):=\left(P_{N} u\right)\left(x, t_{n}\right)-u_{I}^{n}(x), \quad \dot{e}_{N}^{n}(x):=P_{N}\left(\partial_{t} u\right)\left(x, t_{n}\right)-\dot{u}_{I}^{n}(x), \quad x \in \bar{\Omega} .
$$

Using the triangle inequality and noticing the assumption (A), we have

$$
\begin{aligned}
& \left\|e^{n}\right\|_{H^{2}} \leq\left\|u\left(\cdot, t_{n}\right)-P_{N} u\left(\cdot, t_{n}\right)\right\|_{H^{2}}+\left\|e_{N}^{n}\right\|_{H^{2}} \lesssim h^{m_{0}+2}+\left\|e_{N}^{n}\right\|_{H^{2}}, \\
& \left\|\dot{e}^{n}\right\|_{H^{2}} \leq\left\|\partial_{t} u\left(\cdot, t_{n}\right)-P_{N} \partial_{t} u\left(\cdot, t_{n}\right)\right\|_{H^{2}}+\left\|\dot{e}_{N}^{n}\right\|_{H^{2}} \lesssim \frac{1}{\varepsilon^{2}} h^{m_{0}+2}+\left\|\dot{e}_{N}^{n}\right\|_{H^{2}} .
\end{aligned}
$$

Thus we need only obtain estimates for $\left\|e_{N}^{n}\right\|_{H^{2}}$ and $\left\|\dot{e}_{N}^{n}\right\|_{H^{2}}$, which will be done by introducing the following error energy functional

$$
\mathcal{E}\left(e_{N}^{n}, \dot{e}_{N}^{n}\right):=\varepsilon^{2}\left\|\dot{e}_{N}^{n}\right\|_{H^{2}}^{2}+\left\|\partial_{x} e_{N}^{n}\right\|_{H^{2}}^{2}+\frac{1}{\varepsilon^{2}}\left\|e_{N}^{n}\right\|_{H^{2}}^{2}, \quad 0 \leq n \leq \frac{T}{\tau},
$$

and establishing the following several lemmas.

Lemma 4.2 (Formulation of the exact solution). Denote the Fourier expansion of the exact solution $u(x, t)$ of the problem (3.3) as

$$
u(x, t)=\sum_{l=-\infty}^{\infty} \widehat{u}_{l}(t) e^{i \mu_{l}(x-a)}, \quad x \in \bar{\Omega}, \quad t \geq 0,
$$


then we have

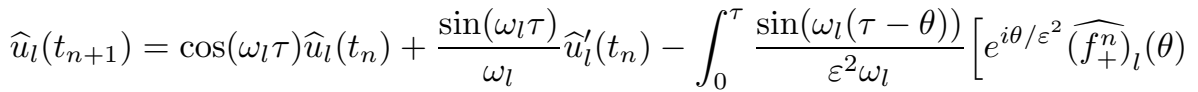

$$
\begin{aligned}
& \left.+e^{-i \theta / \varepsilon^{2}} \widehat{\left(\widehat{f_{-}^{n}}\right)_{l}}(\theta)+e^{3 i \theta / \varepsilon^{2}} \widehat{\left(g_{+}^{n}\right)_{l}}(\theta)+e^{-3 i \theta / \varepsilon^{2}} \widehat{\left(\widehat{g}_{-}^{n}\right)_{l}}(\theta)+{\widehat{\left(w^{n}\right)_{l}}}_{l}(\theta)\right] d \theta
\end{aligned}
$$

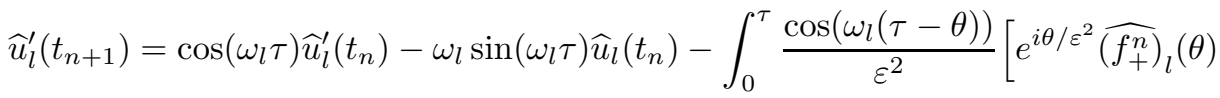

$$
\begin{aligned}
& \left.+e^{-i \theta / \varepsilon^{2}} \widehat{\left(\widehat{f_{-}^{n}}\right)_{l}}(\theta)+e^{3 i \theta / \varepsilon^{2}} \widehat{\left(g_{+}^{n}\right)_{l}}(\theta)+e^{-3 i \theta / \varepsilon^{2}} \widehat{\left(\widehat{g_{-}^{n}}\right)_{l}}(\theta)+\widehat{\left(w^{n}\right)_{l}}(\theta)\right] d \theta .
\end{aligned}
$$

Proof. Substituting (4.10) with $t=t_{n}+s$ into (3.3), we have

$$
\varepsilon^{2} \widehat{u}_{l}^{\prime \prime}\left(t_{n}+s\right)+\left(\mu_{l}^{2}+\frac{1}{\varepsilon^{2}}\right) \widehat{u}_{l}\left(t_{n}+s\right)+\widehat{f(u)_{l}}\left(t_{n}+s\right)=0, \quad s>0 .
$$

Applying the variation-of-constant formula to (4.12) and noticing (3.12), we get

$$
\begin{aligned}
\widehat{u}_{l}\left(t_{n}+s\right)= & \cos \left(\omega_{l} s\right) \widehat{u}_{l}\left(t_{n}\right)+\frac{\sin \left(\omega_{l} s\right)}{\omega_{l}} \widehat{u}_{l}^{\prime}\left(t_{n}\right) \\
& -\int_{0}^{s} \frac{\sin \left(\omega_{l}(s-\theta)\right)}{\varepsilon^{2} \omega_{l}} \widehat{f(u)_{l}}\left(t_{n}+\theta\right) d \theta, \quad 0 \leq s \leq \tau .
\end{aligned}
$$

For the cubic nonlinearity $f(u)=\lambda|u|^{2} u$ and noticing (2.2), (3.1) and (3.2), we have

$$
\begin{aligned}
f\left(u\left(x, t_{n}+s\right)\right)= & e^{i s / \varepsilon^{2}} f_{+}^{n}(x, s)+e^{-i s / \varepsilon^{2}} \overline{f_{-}^{n}}(x, s)+e^{3 i s / \varepsilon^{2}} g_{+}^{n}(x, s) \\
& +e^{-3 i s / \varepsilon^{2}} \overline{g_{-}^{n}}(x, s)+w^{n}(x, s), \quad x \in \bar{\Omega}, \quad 0 \leq s \leq \tau,
\end{aligned}
$$

where

$$
\left\{\begin{array}{l}
f_{ \pm}^{n}(x, s)=f_{ \pm}\left(z_{+}^{n}(x, s), z_{-}^{n}(x, s)\right), \quad g_{ \pm}^{n}(x, s)=g_{ \pm}\left(z_{+}^{n}(x, s), z_{-}^{n}(x, s)\right), \\
w^{n}(x, s)=w^{n}\left(z_{+}^{n}(x, s), z_{-}^{n}(x, s), r^{n}(x, s) ; s\right), \quad x \in \bar{\Omega}, \quad 0 \leq s \leq \tau .
\end{array}\right.
$$

Plugging (4.14) and (4.15) into (4.13), we get

$$
\begin{aligned}
& \widehat{u}_{l}\left(t_{n}+s\right)=\cos \left(\omega_{l} s\right) \widehat{u}_{l}\left(t_{n}\right)+\frac{\sin \left(\omega_{l} s\right)}{\omega_{l}} \widehat{u}_{l}^{\prime}\left(t_{n}\right)-\int_{0}^{s} \frac{\sin \left(\omega_{l}(s-\theta)\right)}{\varepsilon^{2} \omega_{l}}\left[e^{i \theta / \varepsilon^{2}} \widehat{\left(f_{+}^{n}\right)_{l}}(\theta)\right. \\
& \left.+e^{-i \theta / \varepsilon^{2}} \widehat{\left(\widehat{f_{-}^{n}}\right)_{l}}(\theta)+e^{3 i \theta / \varepsilon^{2}}{\widehat{\left(g_{+}^{n}\right)_{l}}}_{l}(\theta)+e^{-3 i \theta / \varepsilon^{2}} \widehat{\left(\widehat{g_{-}^{n}}\right)_{l}}(\theta)+\widehat{\left(w^{n}\right)_{l}}(\theta)\right] d \theta .
\end{aligned}
$$

Then we can obtain (4.11a) by setting $s=\tau$ in (4.16) and get 4.11b) by taking derivative with respect to $s$ in (4.16) and then letting $s=\tau$. $\mathrm{C}$

LEMMA 4.3 (A new formulation of MTI-FP). For $n \geq 0$, expanding $u_{I}^{n}(x)$ and $\dot{u}_{I}^{n}(x)$ in 4.2) into Fourier series as

$$
u_{I}^{n}(x)=\sum_{l=-N / 2}^{N / 2-1} \widetilde{\left(u_{I}^{n}\right)_{l}} e^{i \mu_{l}(x-a)}, \quad \dot{u}_{I}^{n}(x)=\sum_{l=-N / 2}^{N / 2-1} \widetilde{\left(\dot{u}_{I}^{n}\right)_{l}} e^{i \mu_{l}(x-a)}, \quad x \in \bar{\Omega}
$$

then we have

$$
\left\{\begin{array}{l}
\widetilde{\left(u_{I}^{n+1}\right)_{l}}=\cos \left(\omega_{l} \tau\right) \widetilde{\left(u_{I}^{n}\right)_{l}}+\frac{\sin \left(\omega_{l} \tau\right) \widetilde{\left(\dot{u}_{I}^{n}\right)_{l}}-\widetilde{G_{l}^{n}}}{\omega_{l}} \\
\widetilde{\left(\dot{u}_{I}^{n+1}\right)_{l}}=-\omega_{l} \sin \left(\omega_{l} \tau\right) \widetilde{\left(u_{I}^{n}\right)_{l}}+\cos \left(\omega_{l} \tau\right) \widetilde{\left(\dot{u}_{I}^{n}\right)_{l}}-\widetilde{\dot{G}_{l}^{n}}, \quad l=-\frac{N}{2}, \ldots, \frac{N}{2}-1
\end{array}\right.
$$


where

$$
\begin{aligned}
\widetilde{G_{l}^{n}}= & e^{i \tau / \varepsilon^{2}}\left[c_{l}(\tau) \widetilde{\left(f_{+}^{0}\right)_{l}}+d_{l}(\tau) \widetilde{\left.\left(\dot{f}_{+}^{0}\right)_{l}\right]}+e^{-i \tau / \varepsilon^{2}}\left[\overline{c_{l}}(\tau) \widetilde{\left(\widetilde{f_{-}^{0}}\right)_{l}}+\overline{d_{l}}(\tau) \widetilde{\left(\dot{\dot{f}_{-}^{0}}\right)_{l}}\right]\right. \\
& \left.+p_{l}(\tau) \widetilde{\left(g_{+}^{0}\right)_{l}}+q_{l}(\tau) \widetilde{\left(\dot{g}_{+}^{0}\right.}\right)_{l}+\overline{p_{l}}(\tau) \widetilde{\left(\overline{g_{-}^{0}}\right)_{l}}+\overline{q_{l}}(\tau) \widetilde{\left(\widetilde{\dot{g}_{-}^{0}}\right)_{l}}, \\
\widetilde{\dot{G}_{l}^{n}}= & e^{i \tau / \varepsilon^{2}}\left[c_{l}^{\prime}(\tau)+\frac{i}{\varepsilon^{2}} c_{l}(\tau)\right] \widetilde{\left(f_{+}^{0}\right)_{l}}+e^{i \tau / \varepsilon^{2}}\left[d_{l}^{\prime}(\tau)+\frac{i}{\varepsilon^{2}} d_{l}(\tau)\right] \widetilde{\left(\dot{f}_{+}^{0}\right)_{l}} \\
& +e^{-i \tau / \varepsilon^{2}}\left[\overline{c_{l}^{\prime}}(\tau)-\frac{i}{\varepsilon^{2}} \overline{c_{l}}(\tau)\right] \widetilde{\left(\widetilde{f_{-}^{0}}\right)_{l}}+e^{-i \tau / \varepsilon^{2}}\left[\widetilde{d_{l}^{\prime}}(\tau)-\frac{i}{\varepsilon^{2}} \overline{d_{l}}(\tau)\right] \widetilde{\left(\widetilde{\dot{f}_{-}^{0}}\right)_{l}} \\
& +p_{l}^{\prime}(\tau) \widetilde{\left(\widetilde{g_{+}^{0}}\right)_{l}}+q_{l}^{\prime}(\tau) \widetilde{\left(\dot{g}_{+}^{0}\right)_{l}}+\overline{p_{l}^{\prime}}(\tau) \widetilde{\left(\overline{g_{-}^{0}}\right)_{l}}+\overline{q_{l}^{\prime}}(\tau) \widetilde{\left(\widetilde{\dot{g}_{-}^{0}}\right)_{l}}+\frac{\tau}{2 \varepsilon^{2}}\left(\widetilde{w^{n+1}}\right)_{l} .
\end{aligned}
$$

Proof. Combining (3.20), (4.17) and (4.2), we have

$$
\left\{\begin{array}{l}
\widetilde{\left(z_{+}^{0}\right)_{l}}=\frac{1}{2}\left[\widetilde{\left(u_{I}^{n}\right)_{l}}-i \varepsilon^{2}\left(\widetilde{\left.\dot{u}_{I}^{n}\right)_{l}}\right], \quad \widetilde{\left(z_{-}^{0}\right)_{l}}=\frac{1}{2}\left[\widetilde{\left(\widetilde{u_{I}^{n}}\right)_{l}}-i \varepsilon^{2} \widetilde{\left(\overline{\dot{u}}_{I}^{n}\right)_{l}}\right],\right. \\
\widetilde{\left(\dot{r}^{0}\right)_{l}}=-\widetilde{\left(\dot{z}_{+}^{0}\right)_{l}}-\widetilde{\left(\widetilde{\dot{z}_{-}^{0}}\right)}, \quad l=-\frac{N}{2}, \ldots, \frac{N}{2}-1 .
\end{array}\right.
$$

Inserting (4.21) into (3.19) and noticing (3.17), (3.18), (4.17) and (4.2), we get

$$
\begin{aligned}
& \widetilde{\left(u_{I}^{n+1}\right)_{l}}=e^{i \tau / \varepsilon^{2}} \widetilde{\left(z_{+}^{n+1}\right)_{l}}+e^{-i \tau / \varepsilon^{2}} \widetilde{\left(\widetilde{z_{-}^{n+1}}\right)_{l}}+\widetilde{\left(r^{n+1}\right)_{l}} \\
& =\operatorname{Re}\left\{e^{i \tau / \varepsilon^{2}} a_{l}(\tau)\right\} \widetilde{\left(u_{I}^{n}\right)_{l}}+\varepsilon^{2} \operatorname{Im}\left\{e^{i \tau / \varepsilon^{2}} a_{l}(\tau)\right\} \widetilde{\left(\dot{u}_{I}^{n}\right)_{l}}+\varepsilon^{2} e^{i \tau / \varepsilon^{2}} b_{l}(\tau) \widetilde{\left(\dot{z}_{+}^{0}\right)_{l}} \\
& +\varepsilon^{2} e^{-i \tau / \varepsilon^{2}} \overline{b_{l}}(\tau) \widetilde{\left(\widetilde{\dot{z}_{-}^{0}}\right)_{l}}+\frac{\sin \left(\omega_{l} \tau\right)}{\omega_{l}} \widetilde{\left(\dot{r}^{0}\right)_{l}}-\widetilde{G_{l}^{n}}, \quad l=-\frac{N}{2}, \ldots, \frac{N}{2}-1, \\
& \widetilde{\left(\dot{u}_{I}^{n+1}\right)_{l}}=e^{i \tau / \varepsilon^{2}}\left[\widetilde{\left(\dot{z}_{+}^{n+1}\right)_{l}}+\frac{i}{\varepsilon^{2}} \widetilde{\left(z_{+}^{n+1}\right)_{l}}\right]+e^{-i \tau / \varepsilon^{2}}\left[\widetilde{\left(\widetilde{\dot{z}_{-}^{n+1}}\right)_{l}}-\frac{i}{\left.\varepsilon^{2}\left(\widetilde{z_{-}^{n+1}}\right)_{l}\right]}+\widetilde{\left(\dot{r}^{n+1}\right)_{l}}\right. \\
& =\operatorname{Re}\left\{e^{i \tau / \varepsilon^{2}} a_{l}^{\prime}(\tau)+\frac{i}{\varepsilon^{2}} e^{\frac{i \tau}{\varepsilon^{2}}} a_{l}(\tau)\right\} \widetilde{\left(u_{I}^{n}\right)_{l}}+\varepsilon^{2} e^{-i \tau / \varepsilon^{2}}\left[\overline{b_{l}^{\prime}}(\tau)-\frac{i}{\varepsilon^{2}} \overline{b_{l}}(\tau)\right] \widetilde{\left(\widetilde{\dot{z}_{-}^{0}}\right)_{l}} \\
& +\varepsilon^{2} \operatorname{Im}\left\{e^{i \tau / \varepsilon^{2}} a_{l}^{\prime}(\tau)+\frac{i}{\varepsilon^{2}} e^{\frac{i \tau}{\varepsilon^{2}}} a_{l}(\tau)\right\} \widetilde{\left(\dot{u}_{I}^{n}\right)_{l}}+\varepsilon^{2} e^{i \tau / \varepsilon^{2}}\left[b_{l}^{\prime}(\tau)+\frac{i}{\varepsilon^{2}} b_{l}(\tau)\right] \widetilde{\left(\dot{z}_{+}^{0}\right)_{l}} \\
& +\cos \left(\omega_{l} \tau\right) \widetilde{\left(\dot{r}^{0}\right)_{l}}-\widetilde{\dot{G}_{l}^{n}}, \quad l=-\frac{N}{2}, \ldots, \frac{N}{2}-1,
\end{aligned}
$$

where $\operatorname{Re}(\alpha)$ and $\operatorname{Im}(\alpha)$ denote the real and imaginary parts of a complex number $\alpha$, respectively. Thus we can obtain (4.18) from (4.22) by using the fact that $a_{l}(\tau)=$ $a_{-l}(\tau)$ and $b_{l}(\tau)=b_{-l}(\tau)$ for $l=-N / 2, \ldots, N / 2-1$ in (3.12).

For $0 \leq n \leq \frac{T}{\tau}$, let $z_{ \pm}^{n}(x, s)$ and $r^{n}(x, s)$ be the solution of the MDF (3.4)-(3.5) with $\phi_{1}^{n}(x)=u\left(x, t_{n}\right)$ and $\phi_{2}^{n}(x)=\varepsilon^{2} \partial_{t} u\left(x, t_{n}\right)$ for $x \in \bar{\Omega}$, then we have

LEMMA 4.4 (A prior estimate of MDF). Under the assumption (A), there exists a constant $\tau_{1}>0$ independent of $0<\varepsilon \leq 1$ and $h>0$, such that for $0<\tau \leq \tau_{1}$

$$
\begin{aligned}
& \left\|z_{ \pm}^{n}\right\|_{L^{\infty}\left([0, \tau] ; H^{m_{0}+2}\right)}+\left\|\partial_{s} z_{ \pm}^{n}\right\|_{L^{\infty}\left([0, \tau] ; H^{m_{0}+1}\right)}+\left\|\partial_{s s} z_{ \pm}^{n}\right\|_{L^{\infty}\left([0, \tau] ; H^{\left.m_{0}\right)}\right.} \lesssim 1 \\
& \left\|r^{n}\right\|_{L^{\infty}\left([0, \tau] ; H^{4}\right)}+\varepsilon^{2}\left\|\partial_{s} r^{n}\right\|_{L^{\infty}\left([0, \tau] ; H^{3}\right)}+\varepsilon^{4}\left\|\partial_{s s} r^{n}\right\|_{L^{\infty}\left([0, \tau] ; H^{2}\right)} \lesssim \varepsilon^{2}
\end{aligned}
$$


Proof. From (3.5) and noticing the assumption (A) and (3.1), we have

$$
\begin{aligned}
& \left\|z_{ \pm}^{n}(\cdot, 0)\right\|_{H^{m_{0}+4}} \lesssim\left\|u\left(\cdot, t_{n}\right)\right\|_{H^{m_{0}+4}}+\varepsilon^{2}\left\|\partial_{t} u\left(\cdot, t_{n}\right)\right\|_{H^{m_{0}+4}} \lesssim 1 \\
& \left\|\partial_{s} z_{ \pm}^{n}(\cdot, 0)\right\|_{H^{m_{0}+2}} \lesssim\left\|\partial_{x x} z_{ \pm}^{n}(\cdot, 0)\right\|_{H^{m_{0}+2}}+\left\|f_{ \pm}\left(z_{+}^{n}(\cdot, 0), z_{-}^{n}(\cdot, 0)\right)\right\|_{H^{m_{0}+2}} \lesssim 1
\end{aligned}
$$

which immediately imply

$$
\left\|\partial_{s} r^{n}(\cdot, 0)\right\|_{H^{m_{0}+2}} \leq\left\|\partial_{s} z_{+}^{n}(\cdot, 0)\right\|_{H^{m_{0}+2}}+\left\|\partial_{s} z_{-}^{n}(\cdot, 0)\right\|_{H^{m_{0}+2}} \lesssim 1 .
$$

Similar to the proof for the nonlinear Schrödinger equation with wave operator [3, 4, we can easily establish (4.23) and the details are omitted here for brevity. Taking the Fourier expansion of $r^{n}(x, s)$ and noticing (3.4), (3.5), (3.1) and (3.2), we obtain

$$
r^{n}(x, s)=\sum_{l=-\infty}^{\infty} \widehat{\left(r^{n}\right)_{l}}(s) e^{i \mu_{l}(x-a)}, \quad x \in \bar{\Omega}, \quad 0 \leq s \leq \tau,
$$

where for $l \in \mathbb{Z}$

$$
\begin{aligned}
& \widehat{\left(r^{n}\right)_{l}}(s)= \frac{\sin \left(\omega_{l} s\right)}{\omega_{l}}{\widehat{\left(r^{n}\right)_{l}^{\prime}}}_{l}^{\prime}(0)-\int_{0}^{s} \frac{\sin \left(\omega_{l}(s-\theta)\right)}{\varepsilon^{2} \omega_{l}} e^{3 i \theta / \varepsilon^{2}} \widehat{\left(g_{+}^{n}\right)_{l}(\theta) d \theta} \\
&(4.27) \quad-\int_{0}^{s} \frac{\sin \left(\omega_{l}(s-\theta)\right)}{\varepsilon^{2} \omega_{l}} e^{-3 i \theta / \varepsilon^{2}} \widehat{\left(\widehat{\left.g_{-}^{n}\right)_{l}}\right.}(\theta) d \theta-\int_{0}^{s} \frac{\sin \left(\omega_{l}(s-\theta)\right)}{\varepsilon^{2} \omega_{l}} \widehat{\left(w^{n}\right)_{l}}(\theta) d \theta .
\end{aligned}
$$

Let $N_{\varepsilon}:=\left[\frac{b-a}{2 \pi \varepsilon}\right]=O\left(\frac{1}{\varepsilon}\right)$ be the integer part of $\frac{b-a}{2 \pi \varepsilon}$. From (4.27), integrating by parts and using the Cauchy's and Hölder's inequalities, we obtain for $|l| \leq N_{\varepsilon}$

$$
\begin{aligned}
& \left|\widehat{\left(r^{n}\right)_{l}}(s)\right|^{2} \lesssim\left|\varepsilon^{2}\right|{\widehat{\left(r^{n}\right)_{l}}}_{l}^{\prime}(0) \mid+\varepsilon^{2}\left[\left|\widehat{\left(g_{+}^{n}\right)_{l}}(s)\right|+\left|\widehat{\left(g_{+}^{n}\right)_{l}}(0)\right|+\left|\widehat{\left(\widehat{g_{-}^{n}}\right)_{l}}(s)\right|+\left|\widehat{\left(\widehat{g_{-}^{n}}\right)_{l}}(0)\right|\right] \\
& +\left.\int_{0}^{s}\left[\varepsilon^{2}\left(\left|{\widehat{\left(g_{+}^{n}\right)_{l}^{\prime}}}_{l}^{\prime}(\theta)\right|+\left|{\widehat{\left(\bar{g}_{-}^{n}\right.}}_{l}^{\prime}(\theta)\right|\right)+\left|\widehat{\left(w^{n}\right)_{l}}(\theta)\right|\right] d \theta\right|^{2} \\
& \lesssim \varepsilon^{4}\left[\left|{\widehat{\left(r^{n}\right)_{l}}}_{l}^{\prime}(0)\right|^{2}+\left|\widehat{\left(g_{+}^{n}\right)_{l}}(s)\right|^{2}+\left|\widehat{\left(g_{+}^{n}\right)_{l}}(0)\right|^{2}+\left|\widehat{\left(\widehat{g_{-}^{n}}\right)_{l}}(s)\right|^{2}+\left|\widehat{\left(\widehat{g_{-}^{n}}\right)_{l}}(0)\right|^{2}\right] \\
& +\int_{0}^{s}\left[\varepsilon^{4}\left(\left|{\widehat{\left(g_{+}^{n}\right)_{l}}}_{l}^{\prime}(\theta)\right|^{2}+\left|{\widehat{\left(g_{-}^{n}\right.}}_{l}^{\prime}(\theta)\right|^{2}\right)+\left|{\widehat{\left(w^{n}\right)_{l}}}_{l}(\theta)\right|^{2}\right] d \theta \text {. }
\end{aligned}
$$

Here we use the fact that for $|l| \leq N_{\varepsilon}$

$$
\begin{aligned}
& T_{l}(\theta)=\frac{\varepsilon^{2} e^{3 i \theta / \varepsilon^{2}}}{\varepsilon^{4} \omega_{l}^{2}-9}\left[\cos \left(\omega_{l}(s-\theta)\right)+\frac{3 i}{\varepsilon^{2} \omega_{l}} \sin \left(\omega_{l}(s-\theta)\right)\right]=O\left(\varepsilon^{2}\right), \\
& T_{l}^{\prime}(\theta)=\frac{\sin \left(\omega_{l}(s-\theta)\right)}{\varepsilon^{2} \omega_{l}} e^{3 i \theta / \varepsilon^{2}}=O(1), \quad 0 \leq \theta \leq s \leq \tau, \quad 0<\varepsilon \leq 1 .
\end{aligned}
$$

Similarly, we can get for $|l|>N_{\varepsilon}$

$$
\left.\left|\widehat{\left(r^{n}\right)_{l}}(s)\right|^{2} \lesssim \varepsilon^{4}\left|{\widehat{\left(r^{n}\right)_{l}}}_{l}^{\prime}(0)\right|^{2}+\left.\int_{0}^{s}\left[\left|\widehat{\left(g_{+}^{n}\right)_{l}}(\theta)\right|^{2}+\left|\widehat{\left(\widehat{g_{-}^{n}}\right)_{l}}(\theta)\right|^{2}+\mid \widehat{\left(w^{n}\right)}\right)_{l}(\theta)\right|^{2}\right] d \theta .
$$

Multiplying (4.28) and (4.29) by $1+\mu_{l}^{2}+\ldots+\mu_{l}^{8}$, then summing them up for $l \in \mathbb{Z}$, 
we obtain

$$
\begin{aligned}
&\left\|r^{n}(\cdot, s)\right\|_{H^{4}}^{2} \lesssim \sum_{l=-\infty}^{\infty}\left(1+\mu_{l}^{2}+\ldots+\mu_{l}^{8}\right)\left|\widehat{\left(r^{n}\right)_{l}}(s)\right|^{2} \\
& \quad \lesssim \sum_{l=-\infty}^{\infty}\left(\sum_{m=0}^{4} \mu_{l}^{2 m}\right) \int_{0}^{s}\left|\widehat{\left(w^{n}\right)_{l}}(\theta)\right|^{2} d \theta+\varepsilon^{4}\left[\left\|\partial_{s} r^{n}(\cdot, 0)\right\|_{H^{4}}^{2}+\left\|g_{+}^{n}\right\|_{L^{\infty}\left([0, \tau] ; H^{4}\right)}\right. \\
&\left.\quad+\left\|g_{-}^{n}\right\|_{L^{\infty}\left([0, \tau] ; H^{4}\right)}+s\left\|\partial_{s} g_{+}^{n}\right\|_{L^{\infty}\left([0, \tau] ; H^{4}\right)}+s\left\|\partial_{s} g_{-}^{n}\right\|_{L^{\infty}\left([0, \tau] ; H^{4}\right)}\right] \\
& \quad+s\left[\left\|g_{+}^{n}-P_{N_{\varepsilon}} g_{+}^{n}\right\|_{L^{\infty}\left([0, \tau] ; H^{4}\right)}^{2}+\left\|g_{-}^{n}-P_{N_{\varepsilon}} g_{-}^{n}\right\|_{L^{\infty}\left([0, \tau] ; H^{4}\right)}^{2}\right] \\
&(4.30) \lesssim \varepsilon^{4}+\int_{0}^{s}\left\|w^{n}(\cdot, \theta)\right\|_{H^{4}}^{2} d \theta \lesssim \varepsilon^{4}+\int_{0}^{s}\left\|r^{n}(\cdot, \theta)\right\|_{H^{4}}^{2} d \theta, \quad 0 \leq s \leq \tau .
\end{aligned}
$$

Combining (4.30), (4.25), noticing $r^{n}(x, 0) \equiv 0$ for $x \in \bar{\Omega}$, and adapting the standard bootstrap argument for the nonlinear wave equation [40, we have that there exists a positive constant $\tau_{1}>0$ independent of $\varepsilon$ and $h$ such that

$$
\left\|r^{n}\right\|_{L^{\infty}\left([0, \tau] ; H^{4}\right)} \lesssim \varepsilon^{2}
$$

Similarly we can obtain

$$
\left\|\partial_{s} r^{n}\right\|_{L^{\infty}\left([0, \tau] ; H^{3}\right)} \lesssim 1, \quad\left\|\partial_{s s} r^{n}\right\|_{L^{\infty}\left([0, \tau] ; H^{2}\right)} \lesssim \frac{1}{\varepsilon^{2}},
$$

which, together with (4.31), immediately imply the desired inequality (4.24).

Combining the above lemmas and defining the local truncation error as

$$
\xi^{n}(x)=\sum_{l=-N / 2}^{N / 2-1} \widehat{\xi_{l}^{n}} e^{i \mu_{l}(x-a)}, \quad \dot{\xi}^{n}(x)=\sum_{l=-N / 2}^{N / 2-1} \widehat{\dot{\xi}_{l}^{n}} e^{i \mu_{l}(x-a)}, \quad x \in \bar{\Omega},
$$

where

$$
\left\{\begin{array}{l}
\widehat{\xi_{l}^{n}}:=\widehat{u}_{l}\left(t_{n+1}\right)-\left[\cos \left(\omega_{l} \tau\right) \widehat{u}_{l}\left(t_{n}\right)+\frac{\sin \left(\omega_{l} \tau\right)}{\omega_{l}} \widehat{u}_{l}^{\prime}\left(t_{n}\right)-\widehat{\mathcal{G}_{l}^{n}}\right], \\
\widehat{\dot{\xi}_{l}^{n}}:=\widehat{u}_{l}^{\prime}\left(t_{n+1}\right)-\left[-\omega_{l} \sin \left(\omega_{l} \tau\right) \widehat{u}_{l}\left(t_{n}\right)+\cos \left(\omega_{l} \tau\right) \widehat{u}_{l}^{\prime}\left(t_{n}\right)-\widehat{\mathcal{G}_{l}^{n}}\right],
\end{array}\right.
$$

with

$$
\begin{aligned}
& \widehat{\mathcal{G}_{l}^{n}}=e^{i \tau / \varepsilon^{2}}\left[c_{l}(\tau) \widehat{\left(f_{+}^{n}\right)_{l}}(0)+d_{l}(\tau){\widehat{\left(f_{+}^{n}\right)_{l}^{\prime}}}_{l}^{\prime}(0)\right]+e^{-i \tau / \varepsilon^{2}}\left[{\overline{c_{l}}}_{l}(\tau) \widehat{\left(\overline{f-}_{-}^{n}\right.}\right)_{l}(0)+{\overline{d_{l}}}_{(\tau)}\left(\widehat{\left(f_{-}^{n}\right)_{l}^{\prime}}(0)\right] \\
& \left.(4.35 \mathrm{a})+p_{l}(\tau) \widehat{\left(g_{+}^{n}\right.}\right)_{l}(0)+q_{l}(\tau){\widehat{\left(g_{+}^{n}\right.}}_{l}^{\prime}(0)+\overline{p_{l}}(\tau) \widehat{\left(\overline{g_{-}}\right)_{l}}(0)+\overline{q_{l}}(\tau)\left(\widehat{\overline{g-}}_{l}^{\prime}(0),\right. \\
& \widehat{\mathcal{G}_{l}^{n}}=e^{i \tau / \varepsilon^{2}}\left[c_{l}^{\prime}(\tau)+\frac{i}{\varepsilon^{2}} c_{l}(\tau)\right] \widehat{\left(f_{+}^{n}\right)_{l}}(0)+e^{i \tau / \varepsilon^{2}}\left[d_{l}^{\prime}(\tau)+\frac{i}{\varepsilon^{2}} d_{l}(\tau)\right] \widehat{\left(f_{+}^{n}\right)_{l}^{\prime}}(0) \\
& +e^{-i \tau / \varepsilon^{2}}\left[\overline{c_{l}^{\prime}}(\tau)-\frac{i}{\varepsilon^{2}} \overline{c_{l}}(\tau)\right] \widehat{\left(\widehat{f_{-}^{n}}\right)_{l}}(0)+e^{-i \tau / \varepsilon^{2}}\left[\overline{d_{l}^{\prime}}(\tau)-\frac{i}{\varepsilon^{2}}{\overline{d_{l}}}_{l}(\tau)\right]{\widehat{\left(\widehat{f_{-}^{n}}\right.}}_{l}^{\prime}(0) \\
& +p_{l}^{\prime}(\tau) \widehat{\left(g_{+}^{n}\right)_{l}}(0)+q_{l}^{\prime}(\tau){\widehat{\left(g_{+}^{n}\right)_{l}^{\prime}}}_{l}^{\prime}(0)+\overline{p_{l}^{\prime}}(\tau) \widehat{\left(\widehat{g_{-}^{n}}\right)_{l}}(0)+\overline{q_{l}^{\prime}}(\tau) \widehat{\left(\bar{g}_{-}^{n}\right)_{l}^{\prime}}(0)+\frac{\tau}{2 \varepsilon^{2}} \widehat{\left(w^{n}\right)_{l}}(\tau) .
\end{aligned}
$$

Then we have the following estimates for them.

Lemma 4.5 (Estimates on $\xi^{n}$ and $\dot{\xi}^{n}$ ). Under the assumption (A), when $0<\tau \leq$ $\tau_{1}$, we have two independent estimates for $0<\varepsilon \leq 1$

$$
\mathcal{E}\left(\xi^{n}, \dot{\xi}^{n}\right) \lesssim \frac{\tau^{6}}{\varepsilon^{2}}+\tau^{2} \varepsilon^{2} \quad \text { and } \quad \mathcal{E}\left(\xi^{n}, \dot{\xi}^{n}\right) \lesssim \frac{\tau^{6}}{\varepsilon^{6}}, \quad n=0,1, \ldots, \frac{T}{\tau}-1
$$


Proof. Noticing the fact

$$
b_{l}(\tau-\theta) e^{i \tau / \varepsilon^{2}}=\frac{\sin \left(\omega_{l}(\tau-\theta)\right)}{\varepsilon^{2} \omega_{l}} e^{i \theta / \varepsilon^{2}}, \quad 0 \leq \theta \leq \tau,
$$

subtracting (4.34) from (4.11) and then using the Taylor's expansion, we get

$$
\begin{aligned}
& \widehat{\xi_{l}^{n}}=-\int_{0}^{\tau} \frac{\sin \left(\omega_{l}(\tau-\theta)\right)}{\varepsilon^{2} \omega_{l}}\left[\theta ^ { 2 } \left(e^{i \theta / \varepsilon^{2}} \int_{0}^{1}{\widehat{\left(f_{+}^{n}\right.}}_{l}^{\prime \prime}(\theta \rho)(1-\rho) d \rho\right.\right. \\
& \left.+e^{-i \theta / \varepsilon^{2}} \int_{0}^{1} \widehat{\left(\bar{f}_{-}^{n}\right.}\right)_{l}^{\prime \prime}(\theta \rho)(1-\rho) d \rho+e^{3 i \theta / \varepsilon^{2}} \int_{0}^{1}{\widehat{\left(g_{+}^{n}\right)_{l}^{\prime \prime}}}_{l}^{\prime \prime}(\theta \rho)(1-\rho) d \rho \\
& \left.\left.+e^{-3 i \theta / \varepsilon^{2}} \int_{0}^{1}{\widehat{\left(\overline{g_{-}^{n}}\right.}}_{l}^{\prime \prime}(\theta \rho)(1-\rho) d \rho\right)+\widehat{\left(w^{n}\right)_{l}}(\theta)\right] d \theta, \quad l=-\frac{N}{2}, \ldots, \frac{N}{2}-1 .
\end{aligned}
$$

Using the triangle inequality, we obtain

$$
\begin{aligned}
& \left|\widehat{\xi_{l}^{n}}\right| \lesssim \frac{\tau^{2}}{\varepsilon^{2} \omega_{l}} \int_{0}^{\tau}\left[\int_{0}^{1}\left|\widehat{\left(f_{+}^{n}\right)_{l}^{\prime \prime}}(\theta \rho)\right| d \rho+\int_{0}^{1}\left|\widehat{\left(\widehat{f_{-}^{n}}\right)_{l}^{\prime \prime}}(\theta \rho)\right| d \rho+\int_{0}^{1}\left|\widehat{\left(g_{+}^{n}\right)_{l}^{\prime \prime}}(\theta \rho)\right| d \rho\right. \\
& \left.+\int_{0}^{1}\left|\widehat{\left({\overline{g_{-}^{n}}}_{l}^{\prime \prime}\right.}(\theta \rho)\right| d \rho\right] d \theta+\frac{1}{\varepsilon^{2} \omega_{l}} \int_{0}^{\tau}\left|\widehat{\left(w^{n}\right)_{l}}(\theta)\right| d \theta, \quad l=-\frac{N}{2}, \ldots, \frac{N}{2}-1 .
\end{aligned}
$$

Noting $\frac{1}{\varepsilon^{2} \omega_{l}}=\frac{1}{\sqrt{1+\varepsilon^{2} \mu_{l}^{2}}} \leq 1$ for $l=-\frac{N}{2}, \ldots, \frac{N}{2}-1$ and by Lemma 4.4, we get

$$
\begin{aligned}
\left\|\xi^{n}\right\|_{H^{2}}^{2} \lesssim \tau^{6}\left[\left\|\partial_{s s} f_{+}^{n}\right\|_{L^{\infty}\left([0, \tau] ; H^{2}\right)}^{2}+\left\|\partial_{s s} f_{-}^{n}\right\|_{L^{\infty}\left([0, T] ; H^{2}\right)}^{2}+\left\|\partial_{s s} g_{+}^{n}\right\|_{L^{\infty}\left([0, \tau] ; H^{2}\right)}^{2}\right. \\
\left.\quad+\left\|\partial_{s s} g_{-}^{n}\right\|_{L^{\infty}\left([0, \tau] ; H^{2}\right)}^{2}\right]+\tau^{2}\left\|w^{n}\right\|_{L^{\infty}\left([0, \tau] ; H^{2}\right)}^{2} \lesssim \tau^{6}+\tau^{2} \varepsilon^{4}, \quad 0<\tau \leq \tau_{1} .
\end{aligned}
$$

Similarly, noting $\frac{\left|\mu_{l}\right|}{\varepsilon^{2} \omega_{l}}=\frac{\left|\mu_{l}\right|}{\sqrt{1+\varepsilon^{2} \mu_{l}^{2}}} \leq \frac{1}{\varepsilon}$ for $l=-\frac{N}{2}, \ldots, \frac{N}{2}-1$, we obtain

$$
\left\|\partial_{x} \xi^{n}\right\|_{H^{2}}^{2} \lesssim \frac{\tau^{6}}{\varepsilon^{2}}+\tau^{2} \varepsilon^{2} \quad \text { and } \quad\left\|\dot{\xi}^{n}\right\|_{H^{2}}^{2} \lesssim \frac{\tau^{6}}{\varepsilon^{4}}+\tau^{2}, \quad 0<\tau \leq \tau_{1}
$$

Plugging (4.39) and (4.40) into (4.9) with $e_{N}^{n}=\xi^{n}$ and $\dot{e}_{N}^{n}=\dot{\xi}^{n}$, we immediately get the first inequality in (4.36). On the other hand, for $l=-N / 2, \ldots, N / 2-1$, noticing $\widehat{\left(w^{n}\right)_{l}}(0)=0$ and using the error formula of trapezoidal rule for an integral, we get $(4.41)$

$$
\left|\int_{0}^{\tau} \frac{\sin \left(\omega_{l}(\tau-\theta)\right)}{\varepsilon^{2} \omega_{l}} \widehat{\left(w^{n}\right)_{l}}(\theta) d \theta\right| \lesssim \int_{0}^{\tau} \frac{\theta(\tau-\theta)}{\varepsilon^{2} \omega_{l}}\left|\frac{d^{2}}{d \theta^{2}}\left[\sin \left(\omega_{l}(\tau-\theta)\right) \widehat{\left(w^{n}\right)_{l}}(\theta)\right]\right| d \theta .
$$

Combining (4.41) and (4.38), we have

$$
\begin{aligned}
& \left|\widehat{\xi_{l}^{n}}\right| \lesssim \frac{\tau^{2}}{\varepsilon^{2} \omega_{l}} \int_{0}^{\tau}\left[\int_{0}^{1}\left|\widehat{\left(f_{+}^{n}\right)_{l}^{\prime \prime}}(\theta \rho)\right| d \rho+\int_{0}^{1}\left|\widehat{\left(\widehat{f_{-}^{n}}\right)_{l}^{\prime \prime}}(\theta \rho)\right| d \rho+\int_{0}^{1}\left|\widehat{\left(g_{+}^{n}\right)_{l}^{\prime \prime}}(\theta \rho)\right| d \rho\right. \\
& \left.+\int_{0}^{1}\left|\widehat{\left(\bar{g}_{-}^{n}\right)_{l}^{\prime \prime}}(\theta \rho)\right| d \rho+\omega_{l}^{2}\left|{\widehat{\left(w^{n}\right)_{l}}}_{l}(\theta)\right|+\omega_{l}\left|{\widehat{\left(w^{n}\right)_{l}^{\prime}}}_{l}^{\prime}(\theta)\right|+\left|{\widehat{\left(w^{n}\right)_{l}^{\prime \prime}}}^{\prime}(\theta)\right|\right] d \theta .
\end{aligned}
$$


Noting $\omega_{l} \lesssim\left(1+\left|\mu_{l}\right|\right) / \varepsilon^{2}$ for $l=-\frac{N}{2}, \ldots, \frac{N}{2}-1$, we obtain

$$
\begin{aligned}
\left\|\xi^{n}\right\|_{H^{2}}^{2} \lesssim & \tau^{6}\left[\left\|\partial_{s s} f_{+}^{n}\right\|_{L^{\infty}\left([0, \tau] ; H^{2}\right)}^{2}+\left\|\partial_{s s} f_{-}^{n}\right\|_{L^{\infty}\left([0, \tau] ; H^{2}\right)}^{2}+\left\|\partial_{s s} g_{+}^{n}\right\|_{L^{\infty}\left([0, \tau] ; H^{2}\right)}^{2}\right. \\
& +\left\|\partial_{s s} g_{-}^{n}\right\|_{L^{\infty}\left([0, \tau] ; H^{2}\right)}^{2}+\frac{1}{\varepsilon^{8}}\left\|w^{n}\right\|_{L^{\infty}\left([0, \tau] ; H^{4}\right)}^{2}+\frac{1}{\varepsilon^{4}}\left\|\partial_{s} w^{n}\right\|_{L^{\infty}\left([0, \tau] ; H^{3}\right)}^{2} \\
& \left.+\left\|\partial_{s s} w^{n}\right\|_{L^{\infty}\left([0, \tau] ; H^{2}\right)}^{2}\right] \lesssim \frac{\tau^{6}}{\varepsilon^{4}}, \quad 0<\tau \leq \tau_{1} .
\end{aligned}
$$

Similarly, we can get

$$
\left\|\partial_{x} \xi^{n}\right\|_{H^{2}}^{2} \lesssim \frac{\tau^{6}}{\varepsilon^{6}}, \quad\left\|\dot{\xi}^{n}\right\|_{H^{2}}^{2} \lesssim \frac{\tau^{6}}{\varepsilon^{8}}, \quad 0<\tau \leq \tau_{1} .
$$

Again, substituting (4.42) and (4.43) into (4.9) with $e_{N}^{n}=\xi^{n}$ and $\dot{e}_{N}^{n}=\dot{\xi}^{n}$, we immediately get the second inequality in (4.36).

For any $\mathbf{v} \in Y_{N}$, we denote $v_{-1}=v_{N-1}$ and $v_{n+1}=v_{1}$ and then define the difference operators $\delta_{x}^{+} \mathbf{v} \in Y_{N}$ and $\delta_{x}^{2} \mathbf{v} \in Y_{N}$ as

$$
\delta_{x}^{+} \mathbf{v}_{j}=\frac{v_{j+1}-v_{j}}{h}, \quad \delta_{x}^{2} \mathbf{v}_{j}=\frac{v_{j+1}-2 v_{j}+v_{j-1}}{h^{2}}, \quad j=0,1, \ldots, N .
$$

In addition, we define the following norms as $\|\mathbf{v}\|_{Y, 1}^{2}=\|\mathbf{v}\|_{l^{2}}^{2}+\left\|\delta_{x}^{+} \mathbf{v}\right\|_{l^{2}}^{2}$ and $\|\mathbf{v}\|_{Y, 2}^{2}=$ $\|\mathbf{v}\|_{l^{2}}^{2}+\left\|\delta_{x}^{+} \mathbf{v}\right\|_{l^{2}}^{2}+\left\|\delta_{x}^{2} \mathbf{v}\right\|_{l^{2}}^{2}$ and it is easy to see that

$$
\left\|I_{N} \mathbf{v}\right\|_{H^{1}} \lesssim\|\mathbf{v}\|_{Y, 1} \lesssim\left\|I_{N} \mathbf{v}\right\|_{H^{1}}, \quad\left\|I_{N} \mathbf{v}\right\|_{H^{2}} \lesssim\|\mathbf{v}\|_{Y, 2} \lesssim\left\|I_{N} \mathbf{v}\right\|_{H^{2}}, \quad \forall \mathbf{v} \in Y_{N} .
$$

Let $\mathbf{z}_{ \pm}^{0} \in Y_{N}, \dot{\mathbf{z}}_{ \pm}^{0} \in Y_{N}, \mathbf{f}_{ \pm}^{0} \in Y_{N}, \dot{\mathbf{f}}_{ \pm}^{0} \in Y_{N}, \mathbf{g}_{ \pm}^{0} \in Y_{N}$ and $\dot{\mathbf{g}}_{ \pm}^{0} \in Y_{N}$ with $z_{ \pm, j}^{0}$, $\dot{z}_{ \pm, j}^{0}, f_{ \pm, j}^{0}, \dot{f}_{ \pm, j}^{0}, g_{ \pm, j}^{0}$ and $\dot{g}_{ \pm, j}^{0}$, respectively, for $j=0,1, \ldots, N$ be defined in (3.20), and define the following error functions $\mathbf{e}_{z_{ \pm}}^{n} \in Y_{N}, \dot{\mathbf{e}}_{z_{ \pm}}^{n} \in Y_{N}, \mathbf{e}_{f_{ \pm}}^{n} \in Y_{N}, \dot{\mathbf{e}}_{f_{ \pm}}^{n} \in Y_{N}$, $\mathbf{e}_{g_{ \pm}}^{n} \in Y_{N}$ and $\dot{\mathbf{e}}_{g_{ \pm}}^{n} \in Y_{N}$ as

$$
\left\{\begin{array}{lll}
e_{z_{ \pm}, j}^{n}=z_{ \pm}^{n}\left(x_{j}, 0\right)-z_{ \pm, j}^{0}, & \dot{e}_{z_{ \pm}, j}^{n}=\partial_{s} z_{ \pm}^{n}\left(x_{j}, 0\right)-\dot{z}_{ \pm, j}^{0}, & \\
e_{f_{ \pm}, j}^{n}=f_{ \pm}^{n}\left(x_{j}, 0\right)-f_{ \pm, j}^{0}, & \dot{e}_{f_{ \pm}, j}^{n}=\partial_{s} f_{ \pm}^{n}\left(x_{j}, 0\right)-\dot{f}_{ \pm, j}^{0}, & 0 \leq j \leq N, \\
e_{g_{ \pm}, j}^{n}=g_{ \pm}^{n}\left(x_{j}, 0\right)-g_{ \pm, j}^{0}, & \dot{e}_{g_{ \pm}, j}^{n}=\partial_{s} g_{ \pm}^{n}\left(x_{j}, 0\right)-\dot{g}_{ \pm, j}^{0} .
\end{array}\right.
$$

LEMMA 4.6 (Interpolation error). Under the assumption (A) and assume 4.5) holds (which will be proved by induction later), then we have

$$
\begin{aligned}
\left\|I_{N} \mathbf{e}_{f_{ \pm}}^{n}\right\|_{H^{2}}+\left\|I_{N} \mathbf{e}_{g_{ \pm}}^{n}\right\|_{H^{2}} & \lesssim\left\|e_{N}^{n}\right\|_{H^{2}}+\varepsilon^{2}\left\|\dot{e}_{N}^{n}\right\|_{H^{2}}+h^{m_{0}}, \\
\left\|I_{N} \dot{\mathbf{e}}_{f_{ \pm}}^{n}\right\|_{H^{2}}+\left\|I_{N} \dot{\mathbf{e}}_{g_{ \pm}}^{n}\right\|_{H^{2}} & \lesssim \frac{1}{\tau}\left(\left\|e_{N}^{n}\right\|_{H^{2}}+\varepsilon^{2}\left\|\dot{e}_{N}^{n}\right\|_{H^{2}}+h^{m_{0}}+\tau^{2}\right) .
\end{aligned}
$$

Proof. From (4.45), (4.44), (3.20) and (4.15), we have

$$
\begin{aligned}
\left\|I_{N} \mathbf{e}_{f_{ \pm}}^{n}\right\|_{H^{2}} & \lesssim\left\|\mathbf{e}_{f_{ \pm}}^{n}\right\|_{Y, 2} \\
& \leq \int_{0}^{1}\left[\left\|\partial_{z_{+}} f_{ \pm}\left(\mathbf{z}_{+}^{\theta}, \mathbf{z}_{-}^{n}\right) \cdot \mathbf{e}_{z_{+}}^{n}\right\|_{Y, 2}+\left\|\partial_{z_{-}} f_{ \pm}\left(\mathbf{z}_{+}^{0}, \mathbf{z}_{-}^{\theta}\right) \cdot \mathbf{e}_{z_{-}}^{n}\right\|_{Y, 2}\right] d \theta,
\end{aligned}
$$


where $\mathbf{z}_{ \pm}^{\theta} \in Y_{N}$ and $\mathbf{z}_{ \pm}^{n} \in Y_{N}$ are defined as $\mathbf{z}_{ \pm, j}^{\theta}=\theta z_{ \pm}^{n}\left(x_{j}, 0\right)+(1-\theta) z_{ \pm, j}^{0}$ and $\mathbf{z}_{ \pm}^{n}=z_{ \pm, j}^{n}$, respectively, for $j=0,1, \ldots, N$ and $0 \leq \theta \leq 1$. Under the assumption (4.5) and using the Sobolev's inequality, we get

$$
\begin{aligned}
& \int_{0}^{1}\left\|\partial_{z_{+}} f_{ \pm}\left(\mathbf{z}_{+}^{\theta}, \mathbf{z}_{-}^{n}\right) \cdot \mathbf{e}_{z_{+}}^{n}\right\|_{Y, 2} d \theta \lesssim\left\|\mathbf{e}_{z_{+}}^{n}\right\|_{l^{\infty}} \cdot \int_{0}^{1}\left\|\delta_{x}^{2} \partial_{z_{+}} f_{ \pm}\left(\mathbf{z}_{+}^{\theta}, \mathbf{z}_{-}^{n}\right)\right\|_{l^{2}} d \theta \\
& +\left\|\mathbf{e}_{z_{+}}^{n}\right\|_{Y, 1} \cdot \int_{0}^{1}\left\|\delta_{x}^{+} \partial_{z_{+}} f_{ \pm}\left(\mathbf{z}_{+}^{\theta}, \mathbf{z}_{-}^{n}\right)\right\|_{l^{\infty}} d \theta+\left\|\mathbf{e}_{z_{+}}^{n}\right\|_{Y, 2} \cdot \int_{0}^{1}\left\|\partial_{z_{+}} f_{ \pm}\left(\mathbf{z}_{+}^{\theta}, \mathbf{z}_{-}^{n}\right)\right\|_{l^{\infty}} d \theta \\
& \lesssim\left\|\mathbf{e}_{z_{+}}^{n}\right\|_{Y, 2} \cdot
\end{aligned}
$$

Similarly, we have

$$
\int_{0}^{1}\left\|\partial_{z_{-}} f_{ \pm}\left(z_{+}^{0}, \mathbf{z}_{-}^{\theta}\right) \cdot \mathbf{e}_{z_{-}}^{n}\right\|_{Y, 2} d \theta \lesssim\left\|\mathbf{e}_{z_{-}}^{n}\right\|_{Y, 2}
$$

Plugging the above two inequalities into (4.47), we get

$$
\begin{aligned}
\left\|I_{N} \mathbf{e}_{f_{ \pm}}^{n}\right\|_{H^{2}} & \lesssim\left\|\mathbf{e}_{z_{+}}^{n}\right\|_{Y, 2}+\left\|\mathbf{e}_{z_{-}}^{n}\right\|_{Y, 2} \lesssim\left\|I_{N} \mathbf{e}_{z_{+}}^{n}\right\|_{H^{2}}+\left\|I_{N} \mathbf{e}_{z_{-}}^{n}\right\|_{H^{2}} \\
& \lesssim\left\|I_{N} u\left(\cdot, t_{n}\right)-u_{I}^{n}\right\|_{H^{2}}+\varepsilon^{2}\left\|I_{N} \partial_{t} u\left(\cdot, t_{n}\right)-\dot{u}_{I}^{n}\right\|_{H^{2}} \\
& \lesssim\left\|e_{N}^{n}\right\|_{H^{2}}+\varepsilon^{2}\left\|\dot{e}_{N}^{n}\right\|_{H^{2}}+h^{m_{0}} .
\end{aligned}
$$

In addition, combining (2.7) and (3.20), we obtain

$$
\begin{aligned}
\left\|I_{N} \dot{\mathbf{e}}_{f_{ \pm}}^{n}\right\|_{H^{2}} & \lesssim\left\|\dot{\mathbf{e}}_{f_{ \pm}}^{n}\right\|_{Y, 2} \lesssim\left\|I_{N} \mathbf{e}_{z_{+}}^{n}\right\|_{H^{2}}+\left\|I_{N} \mathbf{e}_{z_{-}}^{n}\right\|_{H^{2}}+\left\|I_{N} \dot{\mathbf{e}}_{z_{+}}^{n}\right\|_{H^{2}}+\left\|I_{N} \dot{\mathbf{e}}_{z_{-}}^{n}\right\|_{H^{2}} \\
\lesssim & \left\|e_{N}^{n}\right\|_{H^{2}}+\varepsilon^{2}\left\|\dot{e}_{N}^{n}\right\|_{H^{2}}+h^{m_{0}}+\left\|\partial_{s} z_{+}^{n}(\cdot, 0)-I_{N} \dot{z}_{+}^{0}\right\|_{H^{2}} \\
& \quad+\left\|\partial_{s} z_{-}^{n}(\cdot, 0)-I_{N} \dot{z}_{-}^{0}\right\|_{H^{2}} .
\end{aligned}
$$

Noticing $\partial_{s} z_{ \pm}^{n}(x, 0)=\frac{i}{2}\left[-\partial_{x x} z_{ \pm}^{n}(x, 0)+f_{ \pm}^{n}\left(z_{+}(x, 0), z_{-}(x, 0)\right)\right]$, we have in Fourier space

$$
\begin{aligned}
\left(\widehat{\left.\partial_{s} z_{ \pm}^{n}\right)_{l}}\right. & =\frac{i}{2}\left[\mu_{l}^{2} \widehat{\left(z_{ \pm}^{n}\right)_{l}}+\widehat{\left(f_{ \pm}^{n}\right)_{l}}\right] \\
& =\frac{i}{2}\left[2 \frac{\sin \left(\frac{1}{2} \tau \mu_{l}^{2}\right)}{\tau} \widehat{\left(z_{ \pm}^{n}\right)_{l}}+\widehat{\left(f_{ \pm}^{n}\right)_{l}}\right]+\frac{i \mu_{l}^{2}}{2}\left(1-\frac{\sin \left(\frac{1}{2} \tau \mu_{l}^{2}\right)}{\frac{1}{2} \tau \mu_{l}^{2}}\right) \widehat{\left(z_{ \pm}^{n}\right)_{l}}
\end{aligned}
$$

Since the 'sinc' function $\operatorname{sinc}(s)=\frac{\sin s}{s}$ if $s \neq 0$ and $\operatorname{sinc}(0)=1$ has the property that $\operatorname{sinc}^{\prime}(0)=0$ and all the derivatives of sinc are bounded, we find

$$
\left|1-\frac{\sin \left(\frac{1}{2} \tau \mu_{l}^{2}\right)}{\frac{1}{2} \tau \mu_{l}^{2}}\right|=\left|\operatorname{sinc}(0)-\operatorname{sinc}\left(\frac{1}{2} \tau \mu_{l}^{2}\right)\right| \leq \frac{1}{2} \tau \mu_{l}^{2}\left\|\operatorname{sinc}^{\prime}(\cdot)\right\|_{L^{\infty}} .
$$

Then from (3.20) and Lemma 4.4 we have for small $\tau$,

$$
\left\|\partial_{s} z_{ \pm}^{n}(\cdot, 0)-I_{N} \dot{z}_{ \pm}^{0}\right\|_{H^{2}} \lesssim \frac{1}{\tau}\left(\left\|e_{N}^{n}\right\|_{H^{2}}+\varepsilon^{2}\left\|\dot{e}_{N}^{n}\right\|_{H^{2}}+h^{m_{0}}\right)+\tau\left\|z_{ \pm}^{n}(\cdot, 0)\right\|_{H^{6}} .
$$

Plugging (4.51) into (4.49), we get

$$
\left\|I_{N} \dot{\mathbf{e}}_{f_{ \pm}}^{n}\right\|_{H^{2}} \lesssim \frac{1}{\tau}\left(\left\|e_{N}^{n}\right\|_{H^{2}}+\varepsilon^{2}\left\|\dot{e}_{N}^{n}\right\|_{H^{2}}+h^{m_{0}}+\tau^{2}\right) .
$$


Similarly, we can get the estimate results for $\left\|I_{N} \mathbf{e}_{g_{ \pm}}^{n}\right\|_{H^{2}}$ and $\left\|I_{N} \dot{\mathbf{e}}_{g_{ \pm}}^{n}\right\|_{H^{2}}$. Combining all, we immediately get (4.46). $\mathrm{u}$

Defining the errors from the nonlinear terms as

$$
\eta^{n}(x):=\sum_{l=-N / 2}^{N / 2-1} \widetilde{\eta_{l}^{n}} e^{i \mu_{l}(x-a)}, \quad \dot{\eta}^{n}(x):=\sum_{l=-N / 2}^{N / 2-1} \widetilde{\dot{\eta}_{l}^{n}} e^{i \mu_{l}(x-a)}, \quad x \in \bar{\Omega}, \quad n \geq 0,
$$

where

$$
\widetilde{\eta_{l}^{n}}=\widehat{\mathcal{G}_{l}^{n}}-\widetilde{G_{l}^{n}}, \quad \widetilde{\dot{\eta}_{l}^{n}}=\widehat{\dot{\mathcal{G}}_{l}^{n}}-\widetilde{\dot{G}_{l}^{n}}, \quad l=-\frac{N}{2}, \ldots \frac{N}{2}-1,
$$

then we have

LEMma 4.7 (Estimates on $\eta^{n}$ and $\dot{\eta}^{n}$ ). Under the same assumptions as in Lemma 4.6. we have for any $0<\tau \leq \tau_{1}$,

$$
\mathcal{E}\left(\eta^{n}, \dot{\eta}^{n}\right) \lesssim \tau^{2} \mathcal{E}\left(e_{N}^{n}, \dot{e}_{N}^{n}\right)+\frac{\tau^{2} h^{2 m_{0}}}{\varepsilon^{2}}+\frac{\tau^{6}}{\varepsilon^{2}}, \quad n=0,1, \ldots, \frac{T}{\tau}-1 .
$$

Proof. Denote

$$
\begin{cases}e_{f_{ \pm}}^{n}(x)=f_{ \pm}^{n}(x)-\left(I_{N} \mathbf{f}_{ \pm}^{0}\right)(x), & \dot{e}_{f_{ \pm}}^{n}(x)=\partial_{s} f_{ \pm}^{n}(x)-\left(I_{N} \dot{\mathbf{f}}_{ \pm}^{0}\right)(x), \\ e_{g_{ \pm}}^{n}(x)=f_{ \pm}^{n}(x)-\left(I_{N} \mathbf{f}_{ \pm}^{0}\right)(x), & \dot{e}_{g_{ \pm}}^{n}(x)=\partial_{s} g_{ \pm}^{n}(x)-\left(I_{N} \dot{\mathbf{g}}_{ \pm}^{0}\right)(x), \quad x \in \Omega .\end{cases}
$$

For $l=-N / 2, \ldots, N / 2-1$, from (4.55), (4.53) and (4.35), using the triangle inequality, we have

$$
\begin{aligned}
& \left|\eta_{l}^{n}\right| \leq\left|c_{l}(\tau)\right|\left[\left|\widehat{\left(e_{f_{+}}^{n}\right)_{l}}\right|+\left|\widehat{\left(\widehat{e_{f_{-}}^{n}}\right)_{l}}\right|\right]+\left|d_{l}(\tau)\right|\left[\left|\widehat{\left(\dot{e}_{f_{+}}^{n}\right)_{l}}\right|+\left|\widehat{\left({\dot{\dot{e}_{f_{-}}^{n}}}_{l} \mid\right.}\right|\right] \\
& +\left|p_{l}(\tau)\right|\left[\left|\widehat{\left(e_{g_{+}}^{n}\right)_{l}}\right|+\left|\widehat{\left(\widehat{e_{g_{-}}^{n}}\right)_{l}}\right|\right]+\left|q_{l}(\tau)\right|\left[\left|\widehat{\left(\hat{e}_{g_{+}}^{n}\right)_{l}}\right|+\left|\widehat{\left(\widehat{\dot{e}_{g_{-}}^{n}}\right)_{l}}\right|\right] \text {. }
\end{aligned}
$$

From (3.16) directly, we have

$$
\begin{aligned}
\left|c_{l}(\tau)\right|+\left|p_{l}(\tau)\right| \lesssim \frac{\tau}{\sqrt{1+\mu_{l}^{2} \varepsilon^{2}}} \lesssim \tau, & \mu_{l}\left(\left|c_{l}(\tau)\right|+\left|p_{l}(\tau)\right|\right) \lesssim \frac{\tau}{\varepsilon}, \\
\left|d_{l}(\tau)\right|+\left|q_{l}(\tau)\right| \lesssim \frac{\tau^{2}}{\sqrt{1+\mu_{l}^{2} \varepsilon^{2}}} \lesssim \tau^{2}, & \mu_{l}\left(\left|d_{l}(\tau)\right|+\left|q_{l}(\tau)\right|\right) \lesssim \frac{\tau^{2}}{\varepsilon} .
\end{aligned}
$$

Inserting (4.57) into (4.56) and using the Cauchy's inequality, we obtain

$$
\begin{aligned}
\left\|\eta^{n}\right\|_{H^{2}}^{2} \lesssim & \tau^{2}\left[\left\|P_{N} e_{f_{+}}^{n}\right\|_{H^{2}}^{2}+\left\|P_{N} e_{f_{-}}^{n}\right\|_{H^{2}}^{2}+\left\|P_{N} e_{g_{+}}^{n}\right\|_{H^{2}}^{2}+\left\|P_{N} e_{g_{-}}^{n}\right\|_{H^{2}}^{2}\right] \\
& +\tau^{4}\left[\left\|P_{N} \dot{e}_{f_{+}}^{n}\right\|_{H^{2}}^{2}+\left\|P_{N} \dot{e}_{f_{-}}^{n}\right\|_{H^{2}}^{2}+\left\|P_{N} \dot{e}_{g_{+}}^{n}\right\|_{H^{2}}^{2}+\left\|P_{N} \dot{e}_{g_{-}}^{n}\right\|_{H^{2}}^{2}\right] \\
\lesssim & \tau^{2}\left[\left\|I_{N} e_{f_{+}}^{n}\right\|_{H^{2}}^{2}+\left\|I_{N} e_{f_{-}}^{n}\right\|_{H^{2}}^{2}+\left\|I_{N} e_{g_{+}}^{n}\right\|_{H^{2}}^{2}+\left\|I_{N} e_{g_{-}}^{n}\right\|_{H^{2}}^{2}\right] \\
& +\tau^{4}\left[\left\|I_{N} \dot{e}_{f_{+}}^{n}\right\|_{H^{2}}^{2}+\left\|I_{N} \dot{e}_{f_{-}}^{n}\right\|_{H^{2}}^{2}+\left\|I_{N} \dot{e}_{g_{+}}^{n}\right\|_{H^{2}}^{2}+\left\|I_{N} \dot{e}_{g_{-}}^{n}\right\|_{H^{2}}^{2}\right]+\tau^{2} h^{2 m_{0}} \\
\lesssim & \tau^{2}\left\|e_{N}^{n}\right\|_{H^{2}}^{2}+\tau^{2} \varepsilon^{4}\left\|\dot{e}_{N}^{n}\right\|_{H^{2}}^{2}+\tau^{2} h^{2 m_{0}}+\tau^{6},
\end{aligned}
$$


and

$$
\left\|\partial_{x} \eta^{n}\right\|_{H^{2}}^{2} \lesssim \frac{\tau^{2}}{\varepsilon^{2}}\left\|e_{N}^{n}\right\|_{H^{2}}^{2}+\tau^{2} \varepsilon^{2}\left\|\dot{e}_{N}^{n}\right\|_{H^{2}}^{2}+\frac{\tau^{2} h^{2 m_{0}}}{\varepsilon^{2}}+\frac{\tau^{6}}{\varepsilon^{2}}
$$

Similarly,

$$
\varepsilon^{2}\left\|\dot{\eta}^{n}\right\|_{H^{2}}^{2} \lesssim \frac{\tau^{2}}{\varepsilon^{2}}\left\|e_{N}^{n}\right\|_{H^{2}}^{2}+\tau^{2} \varepsilon^{2}\left\|\dot{e}_{N}^{n}\right\|_{H^{2}}^{2}+\frac{\tau^{2} h^{2 m_{0}}}{\varepsilon^{2}}+\frac{\tau^{6}}{\varepsilon^{2}} .
$$

Combining (4.58), (4.60) and (4.9) we immediately obtain (4.54).

Proof of Theorem 4.1. The proof will be proceeded by the method of mathematical induction and the energy method. For $n=0$, from the initial data in the MTI-FP (3.17)-(3.20) method and noticing the assumption (A), we have

$$
\left\|e^{0}\right\|_{H^{2}}+\varepsilon^{2}\left\|\dot{e}^{0}\right\|_{H^{2}}=\left\|\phi_{1}-I_{N} \phi_{1}\right\|_{H^{2}}+\left\|\phi_{2}-I_{N} \phi_{2}\right\|_{H^{2}} \lesssim h^{m_{0}+2} \lesssim h^{m_{0}} .
$$

In addition, using the triangle inequality, we know that there exists $h_{1}>0$ independent of $\varepsilon$ such that for $0<h \leq h_{1}$ and $\tau>0$

$$
\left\|u_{I}^{0}\right\|_{H^{2}} \leq\left\|\phi_{1}\right\|_{H^{2}}+\left\|e^{0}\right\|_{H^{2}} \leq C_{0}+1, \quad\left\|\dot{u}_{I}^{0}\right\|_{H^{2}} \leq \frac{\left\|\phi_{2}\right\|_{H^{2}}}{\varepsilon^{2}}+\left\|\dot{e}^{0}\right\|_{H^{2}} \leq \frac{C_{0}+1}{\varepsilon^{2}} .
$$

Thus (4.4)-(4.5) are valid for $n=0$. Now we assume that (4.4)-(4.5) are valid for $0 \leq n \leq m-1 \leq T / \tau-1$. Substracting (4.11) from (4.18), we have

$$
\begin{aligned}
& \widehat{\left(e^{n+1}\right)_{l}}=\widehat{u}_{l}\left(t_{n+1}\right)-\widehat{\left(u_{I}^{n+1}\right)_{l}}=\cos \left(\omega_{l} \tau\right) \widehat{\left(e^{n}\right)_{l}}+\frac{\sin \left(\omega_{l} \tau\right)}{\omega_{l}} \widehat{\left(\dot{e}^{n}\right)_{l}}+\widehat{\xi_{l}^{n}}-\widetilde{\eta_{l}^{n}}, \\
& \widehat{\left(\dot{e}^{n+1}\right)_{l}}=\widehat{u}_{l}^{\prime}\left(t_{n+1}\right)-\widehat{\left(\dot{u}_{I}^{n+1}\right)_{l}}=-\omega_{l} \sin \left(\omega_{l} \tau\right) \widehat{\left(e^{n}\right)_{l}}+\cos \left(\omega_{l} \tau\right) \widehat{\left(\dot{e}^{n}\right)_{l}}+\widehat{\dot{\xi}_{l}^{n}}-\widetilde{\dot{\eta}_{l}^{n}} .
\end{aligned}
$$

Using the Cauchy's inequality, we obtain

$$
\begin{aligned}
& \left|\widehat{\left(e^{n+1}\right)_{l}}\right|^{2} \leq(1+\tau)\left|\cos \left(\omega_{l} \tau\right) \widehat{\left(e^{n}\right)_{l}}+\frac{\sin \left(\omega_{l} \tau\right)}{\omega_{l}} \widehat{\left(\dot{e}^{n}\right)_{l}}\right|^{2}+\frac{1+\tau}{\tau}\left|\widehat{\xi_{l}^{n}}-\widetilde{\eta_{l}^{n}}\right|^{2} \\
& \left|\widehat{\left(\dot{e}^{n+1}\right)_{l}}\right|^{2} \leq(1+\tau)\left|\cos \left(\omega_{l} \tau\right) \widehat{\left(\dot{e}^{n}\right)_{l}}-\omega_{l} \sin \left(\omega_{l} \tau\right) \widehat{\left(e^{n}\right)_{l}}\right|^{2}+\frac{1+\tau}{\tau}\left|\widehat{\dot{\xi}_{l}^{n}}-\widetilde{\dot{\eta}_{l}^{n}}\right|^{2}
\end{aligned}
$$

Multiplying (4.62a) and (4.62b) by $\left(\mu_{l}^{2}+\frac{1}{\varepsilon^{2}}\right)\left(1+\mu_{l}^{2}+\mu_{l}^{4}\right)$ and $\varepsilon^{2}\left(1+\mu_{l}^{2}+\mu_{l}^{4}\right)$, respectively, and then summing them up for $l=-N / 2, \ldots, N / 2-1$, we obtain

$$
\mathcal{E}\left(e_{N}^{n+1}, \dot{e}_{N}^{n+1}\right) \leq(1+\tau) \mathcal{E}\left(e_{N}^{n}, \dot{e}_{N}^{n}\right)+\frac{1+\tau}{\tau} \mathcal{E}\left(\xi^{n}-\eta^{n}, \dot{\xi}^{n}-\dot{\eta}^{n}\right) .
$$

Using the Cauchy's inequality, we get

$$
\mathcal{E}\left(e_{N}^{n+1}, \dot{e}_{N}^{n+1}\right)-\mathcal{E}\left(e_{N}^{n}, \dot{e}_{N}^{n}\right) \lesssim \tau \mathcal{E}\left(e_{N}^{n}, \dot{e}_{N}^{n}\right)+\frac{1+\tau}{\tau}\left[\mathcal{E}\left(\xi^{n}, \dot{\xi}^{n}\right)+\mathcal{E}\left(\eta^{n}, \dot{\eta}^{n}\right)\right]
$$

Inserting (4.54) and the second inequality in (4.36) into (4.63), we get

$$
\mathcal{E}\left(e_{N}^{n+1}, \dot{e}_{N}^{n+1}\right)-\mathcal{E}\left(e_{N}^{n}, \dot{e}_{N}^{n}\right) \lesssim \tau \mathcal{E}\left(e_{N}^{n}, \dot{e}_{N}^{n}\right)+\frac{\tau h^{2 m_{0}}}{\varepsilon^{2}}+\frac{\tau^{5}}{\varepsilon^{6}} .
$$


Summing the above inequality for $0 \leq n \leq m-1$ and then applying the discrete Gronwall's inequality, we have

$$
\mathcal{E}\left(e_{N}^{m}, \dot{e}_{N}^{m}\right) \lesssim \frac{h^{2 m_{0}}}{\varepsilon^{2}}+\frac{\tau^{4}}{\varepsilon^{6}}
$$

Similarly, by using the first inequality in (4.36), we obtain

$$
\mathcal{E}\left(e_{N}^{m}, \dot{e}_{N}^{m}\right) \lesssim \frac{h^{2 m_{0}}}{\varepsilon^{2}}+\frac{\tau^{4}}{\varepsilon^{2}}+\varepsilon^{2}
$$

Combining (4.9), (4.8), (4.64) and (4.65), we get that (4.4) is valid for $n=m$, which implies [12,25]

$$
\left\|e^{m}\right\|_{H^{2}}+\varepsilon^{2}\left\|\dot{e}^{m}\right\|_{H^{2}} \leq h^{m_{0}}+\tau .
$$

Using the triangle inequality, we obtain that these exist $h_{2}>0$ and $\tau_{2}>0$ independent of $\varepsilon$ such that

$$
\begin{aligned}
& \left\|u_{I}^{m}\right\|_{H^{2}} \leq\left\|u\left(\cdot, t_{m}\right)\right\|_{H^{2}}+\left\|\dot{e}^{m}\right\|_{H^{2}} \leq C_{0}+1, \\
& \left\|\dot{u}_{I}^{m}\right\|_{H^{2}} \leq\left\|\partial_{t} u\left(\cdot, t_{m}\right)\right\|_{H^{2}}+\left\|\dot{e}^{m}\right\|_{H^{2}} \leq \frac{C_{0}+1}{\varepsilon^{2}}, \quad 0<h \leq h_{2}, \quad 0<\tau \leq \tau_{2} .
\end{aligned}
$$

Thus (4.5) is also valid for $n=m$. Then the proof is completed by chosen $\tau_{0}=$ $\min \left\{\tau_{1}, \tau_{2}\right\}$ and $h_{0}=\min \left\{h_{1}, h_{2}\right\}$.

REMARK 4.1. Here we emphasize that Theorem 4.1 holds in $2 D$ and $3 D$ and the above approach can be directly extended to the higher dimensions without any extra efforts. The only thing needs to be taken care of is the Sobolev inequality used in Lemma 4.6 in $2 D$ and $3 D$,

$$
\begin{aligned}
& \|u\|_{L^{\infty}(\Omega)} \leq C\|u\|_{H^{2}(\Omega)}, \quad \text { in } 2 D \text { and } 3 D, \\
& \|u\|_{W^{1, p}(\Omega)} \leq C\|u\|_{H^{2}(\Omega)}, \quad 1<p<6 \text { in } 2 D \text { and } 3 D,
\end{aligned}
$$

where $\Omega$ is a bounded domain in $2 D$ or 3D. By using assumption (4.5), Lemma 4.6 will still hold in $2 D$ and $3 D$. (4.5) and error bounds can be proved by induction since our scheme is explicit.

Under a weaker assumption of the regularity

$$
\text { (B) } u \in C^{1}\left([0, T] ; H_{p}^{m_{0}+3}(\Omega)\right), \quad\|u\|_{L^{\infty}\left([0, T] ; H^{m_{0}+3}\right)}+\varepsilon^{2}\left\|\partial_{t} u\right\|_{L^{\infty}\left([0, T] ; H^{m_{0}+3}\right)} \lesssim 1,
$$

with $m_{0} \geq 2$, we can have the $H^{1}$-error estimates of the MTI-FP method by a very similar proof with all the $H^{2}$-norms in above changed into $H^{1}$-norms.

THEOREM 4.8. Under the assumption (B), there exist two constants $0<h_{0} \leq 1$ and $0<\tau_{0} \leq 1$ sufficiently small and independent of $\varepsilon$ such that, for any $0<\varepsilon \leq 1$, when $0<h \leq h_{0}$ and $0<\tau \leq \tau_{0}$, we have

$$
\begin{aligned}
& \left\|e^{n}\right\|_{H^{1}}+\varepsilon^{2}\left\|\dot{e}^{n}\right\|_{H^{1}} \lesssim h^{m_{0}}+\frac{\tau^{2}}{\varepsilon^{2}}, \quad\left\|e^{n}\right\|_{H^{1}}+\varepsilon^{2}\left\|\dot{e}^{n}\right\|_{H^{1}} \lesssim h^{m_{0}}+\tau^{2}+\varepsilon^{2}, \\
& \left\|u^{n}\right\|_{l^{\infty}} \leq C_{0}+1, \quad\left\|\dot{u}^{n}\right\|_{l^{\infty}} \leq \frac{C_{0}+1}{\varepsilon^{2}}, \quad 0 \leq n \leq \frac{T}{\tau}
\end{aligned}
$$

REMARK 4.2. In $1 D$ case, Theorem 4.8 holds without any CFL-type conditions. However for higher dimensional cases, i.e. $d=2$ or $d=3$, due to the use of inverse 
inequality to provide the $l^{\infty}$ control of the numerical solution [2], one has to impose the technical condition

$$
\tau \lesssim \rho_{d}(h), \quad \text { with } \quad \rho_{d}(h)= \begin{cases}1 /|\ln h|, & d=2, \\ \sqrt{h}, & d=3 .\end{cases}
$$

If the solution of the $K G$ is smooth enough, we can always turn to Theorem 4.1 and such CFL type conditions are unnecessary.

REMARK 4.3. If the periodic boundary condition for the $K G$ equation (3.3) is replaced by the homogeneous Dirichlet or Neumann boundary condition, then the MTIFP method and its error estimates are still valid provided that the Fourier basis is replaced by sine or cosine basis.

REMARK 4.4. If the cubic nonlinearity in the $K G$ equation (1.1) is replaced by a general gauge invariant nonlinearity, the general MTI-FP method can be designed similar to those in [6].

5. Numerical results. In this section, we present numerical results of the MTIFP method to confirm our error estimates. In order to do so, we take $d=1$ and $f(u)=|u|^{2} u$ in (1.1) and choose the initial data as

$$
\phi_{1}(x)=(1+i) e^{-x^{2} / 2}, \quad \phi_{2}(x)=\frac{3 e^{-x^{2} / 2}}{2 \varepsilon^{2}}, \quad x \in \mathbb{R} .
$$

The problem is solved on a bounded interval $\Omega=[-16,16]$, i.e. $b=-a=16$, which is large enough to guarantee that the periodic boundary condition does not introduce a significant aliasing error relative to the original problem. To quantify the error, we introduce two error functions:

$$
e_{\varepsilon}^{\tau, h}(T):=\left\|u(\cdot, T=M \tau)-u_{I}^{M}\right\|_{H^{2}}, \quad e_{\infty}^{\tau, h}(T):=\max _{\varepsilon}\left\{e_{\varepsilon}^{\tau, h}(T)\right\} .
$$

Since the analytical solution to this problem is not available, so the 'exact' solution is obtained numerically by the MTI-FP method (3.17)-(3.20) with very fine mesh $h=1 / 32$ and time step $\tau=5 \times 10^{-6}$. Tab. 5.1 shows the spatial error of MTI-FP method at $T=1$ under different $\varepsilon$ and $h$ with a very small time step $\tau=5 \times 10^{-6}$ such that the discretization error in time is negligible. Tab. 5.2 shows the temporal error of MTI-FP method at $T=1$ under different $\varepsilon$ and $\tau$ with a small mesh size $h=1 / 8$ such that the discretization error in space is negligible.

From Tabs. 5.15 .2 and extensive additional results not shown here for brevity, we can draw the following observations:

(i) The MTI-FP method is spectrally accurate in space, which is uniformly for $0<\varepsilon \leq 1$ (cf. Tab. [5.1).

(ii) The MTI-FP method converges uniformly and linearly in time for $\varepsilon \in(0, \tau]$ (cf. last row in Tab. 5.2). In addition, for each fixed $\varepsilon=\varepsilon_{0}>0$, when $\tau$ is small enough, it converges quadratically in time (cf. each row in the upper triangle of Tab. 5.2); and for each fixed $\varepsilon$ small enough, when $\tau$ satisfies $0<\varepsilon<\tau$, it also converges quadratically in time (cf. each row in the lower triangle of Tab. [5.2).

(iii) The MTI-FP method is uniformly accurate for all $\varepsilon \in(0,1]$ under the mesh strategy (or $\varepsilon$-scalability) $\tau=O(1)$ and $h=O(1)$.

6. Conclusions. A MTI-FP method was proposed and analyzed for solving the KG equation with a dimensionless parameter $0<\varepsilon \leq 1$ which is inversely proportional to the speed of light. The key ideas for designing the MTI-FP method are based on (i) 
Table 5.1: Spatial error analysis: $e_{\varepsilon}^{\tau, h}(T=1)$ with $\tau=5 \times 10^{-6}$ for different $\varepsilon$ and $h$.

\begin{tabular}{lllll}
\hline$e_{\varepsilon}^{\tau, h}(T)$ & $h_{0}=1$ & $h_{0} / 2$ & $h_{0} / 4$ & $h_{0} / 8$ \\
\hline$\varepsilon_{0}=0.5$ & $1.65 \mathrm{E}-1$ & $3.60 \mathrm{E}-3$ & $1.03 \mathrm{E}-6$ & $7.34 \mathrm{E}-11$ \\
$\varepsilon_{0} / 2^{1}$ & $2.65 \mathrm{E}-1$ & $9.70 \mathrm{E}-3$ & $9.07 \mathrm{E}-7$ & $5.03 \mathrm{E}-11$ \\
$\varepsilon_{0} / 2^{2}$ & $9.02 \mathrm{E}-1$ & $1.34 \mathrm{E}-2$ & $1.73 \mathrm{E}-7$ & $4.60 \mathrm{E}-11$ \\
$\varepsilon_{0} / 2^{3}$ & $1.13 \mathrm{E}+0$ & $2.98 \mathrm{E}-2$ & $2.25 \mathrm{E}-7$ & $4.10 \mathrm{E}-11$ \\
$\varepsilon_{0} / 2^{4}$ & $4.67 \mathrm{E}-1$ & $3.14 \mathrm{E}-2$ & $1.79 \mathrm{E}-7$ & $4.78 \mathrm{E}-11$ \\
$\varepsilon_{0} / 2^{5}$ & $7.41 \mathrm{E}-1$ & $2.73 \mathrm{E}-2$ & $2.50 \mathrm{E}-7$ & $5.49 \mathrm{E}-11$ \\
$\varepsilon_{0} / 2^{7}$ & $7.41 \mathrm{E}-1$ & $2.62 \mathrm{E}-2$ & $2.12 \mathrm{E}-7$ & $4.96 \mathrm{E}-11$ \\
$\varepsilon_{0} / 2^{9}$ & $6.33 \mathrm{E}-1$ & $3.57 \mathrm{E}-2$ & $1.92 \mathrm{E}-7$ & $5.04 \mathrm{E}-11$ \\
$\varepsilon_{0} / 2^{11}$ & $9.19 \mathrm{E}-1$ & $2.44 \mathrm{E}-2$ & $2.19 \mathrm{E}-7$ & $6.18 \mathrm{E}-11$ \\
$\varepsilon_{0} / 2^{13}$ & $1.18 \mathrm{E}+0$ & $2.38 \mathrm{E}-2$ & $2.59 \mathrm{E}-7$ & $5.86 \mathrm{E}-11$ \\
\hline
\end{tabular}

Table 5.2: Temporal error analysis: $e_{\varepsilon}^{\tau, h}(T=1)$ a nd $e_{\infty}^{\tau, h}(T=1)$ with $h=1 / 8$ for different $\varepsilon$ and $\tau$.

\begin{tabular}{llllllll}
\hline$e_{\varepsilon}^{\tau, h}(T)$ & $\tau_{0}=0.2$ & $\tau_{0} / 2^{2}$ & $\tau_{0} / 2^{4}$ & $\tau_{0} / 2^{6}$ & $\tau_{0} / 2^{8}$ & $\tau_{0} / 2^{10}$ & $\tau_{0} / 2^{12}$ \\
\hline$\varepsilon_{0}=0.5$ & $7.17 \mathrm{E}-1$ & $5.72 \mathrm{E}-2$ & $3.50 \mathrm{E}-3$ & $2.14 \mathrm{E}-4$ & $1.33 \mathrm{E}-5$ & $8.14 \mathrm{E}-7$ & $3.67 \mathrm{E}-8$ \\
rate & - & 1.82 & 2.02 & 2.01 & 2.00 & 2.01 & 2.20 \\
\hline$\varepsilon_{0} / 2^{1}$ & $5.40 \mathrm{E}-1$ & $1.58 \mathrm{E}-1$ & $1.12 \mathrm{E}-2$ & $6.74 \mathrm{E}-4$ & $4.15 \mathrm{E}-5$ & $2.54 \mathrm{E}-6$ & $1.18 \mathrm{E}-7$ \\
rate & - & 0.89 & 1.91 & 2.02 & 2.01 & 2.01 & 2.21 \\
\hline$\varepsilon_{0} / 2^{2}$ & $5.23 \mathrm{E}-1$ & $1.47 \mathrm{E}-1$ & $3.70 \mathrm{E}-2$ & $2.70 \mathrm{E}-3$ & $1.62 \mathrm{E}-4$ & $9.87 \mathrm{E}-6$ & $4.62 \mathrm{E}-7$ \\
rate & - & 0.91 & 0.99 & 1.90 & 2.02 & 2.01 & 2.20 \\
\hline$\varepsilon_{0} / 2^{3}$ & $6.30 \mathrm{E}-1$ & $6.28 \mathrm{E}-2$ & $4.13 \mathrm{E}-2$ & $8.90 \mathrm{E}-3$ & $6.51 \mathrm{E}-4$ & $3.92 \mathrm{E}-5$ & $1.82 \mathrm{E}-6$ \\
rate & - & 1.66 & 0.30 & 1.11 & 1.89 & 2.02 & 2.21 \\
\hline$\varepsilon_{0} / 2^{4}$ & $6.11 \mathrm{E}-1$ & $3.00 \mathrm{E}-2$ & $1.16 \mathrm{E}-2$ & $1.05 \mathrm{E}-2$ & $2.20 \mathrm{E}-3$ & $1.60 \mathrm{E}-4$ & $7.41 \mathrm{E}-6$ \\
rate & - & 2.17 & 0.68 & 0.07 & 1.13 & 1.89 & 2.21 \\
\hline$\varepsilon_{0} / 2^{5}$ & $6.17 \mathrm{E}-1$ & $3.01 \mathrm{E}-2$ & $2.70 \mathrm{E}-3$ & $2.90 \mathrm{E}-3$ & $2.80 \mathrm{E}-3$ & $5.26 \mathrm{E}-4$ & $2.98 \mathrm{E}-5$ \\
rate & - & 2.17 & 1.75 & -0.04 & 0.02 & 1.17 & 2.07 \\
\hline$\varepsilon_{0} / 2^{7}$ & $6.16 \mathrm{E}-1$ & $2.90 \mathrm{E}-2$ & $1.80 \mathrm{E}-3$ & $2.37 \mathrm{E}-4$ & $1.37 \mathrm{E}-4$ & $1.96 \mathrm{E}-4$ & $1.91 \mathrm{E}-4$ \\
rate & - & 2.20 & 2.01 & 1.46 & 0.40 & -0.26 & 0.02 \\
\hline$\varepsilon_{0} / 2^{9}$ & $6.13 \mathrm{E}-1$ & $2.90 \mathrm{E}-2$ & $1.69 \mathrm{E}-3$ & $1.12 \mathrm{E}-4$ & $1.09 \mathrm{E}-5$ & $5.51 \mathrm{E}-6$ & $1.69 \mathrm{E}-6$ \\
rate & - & 2.20 & 2.03 & 1.96 & 1.68 & 0.49 & 0.85 \\
\hline$\varepsilon_{0} / 2^{11}$ & $6.16 \mathrm{E}-1$ & $2.90 \mathrm{E}-2$ & $1.69 \mathrm{E}-3$ & $1.05 \mathrm{E}-4$ & $6.95 \mathrm{E}-6$ & $9.97 \mathrm{E}-7$ & $3.38 \mathrm{E}-7$ \\
rate & - & 2.20 & 2.03 & 2.00 & 1.96 & 1.40 & 0.78 \\
\hline$\varepsilon_{0} / 2^{13}$ & $6.20 \mathrm{E}-1$ & $2.92 \mathrm{E}-2$ & $1.69 \mathrm{E}-3$ & $1.06 \mathrm{E}-4$ & $6.61 \mathrm{E}-6$ & $3.94 \mathrm{E}-7$ & $2.38 \mathrm{E}-8$ \\
rate & - & 2.20 & 2.04 & 2.00 & 2.00 & 2.03 & 2.02 \\
\hline \hline$e_{\infty}^{\tau, h}(T)$ & $7.17 \mathrm{E}-1$ & $1.58 \mathrm{E}-1$ & $4.13 \mathrm{E}-2$ & $1.05 \mathrm{E}-2$ & $2.80 \mathrm{E}-3$ & $5.26 \mathrm{E}-4$ & $1.91 \mathrm{E}-4$ \\
rate & - & 1.09 & 0.97 & 0.99 & 1.00 & 1.15 & 0.74 \\
\hline
\end{tabular}

carrying out a multiscale decomposition by frequency at each time step with proper choice of transmission conditions between time steps, and (ii) adapting the Fourier spectral for spatial discretization and the EWI for integrating second-order highly oscillating ODEs. Rigorous error bounds for the MTI-FP method were established, which imply that the MTI-FP method converges uniformly and optimally in space with spectral convergence rate, and uniformly in time with linear convergence rate for 
$\varepsilon \in(0,1]$ and optimally with quadratic convergence rate in the regimes when either $\varepsilon=O(1)$ or $0<\varepsilon \leq \tau$. Numerical results confirmed these error bounds and suggested that they are sharp.

\section{REFERENCES}

[1] R. Adams, Sobolev Spaces, Academic Press, Boston, 1975.

[2] W. BAO, Y. CAI, Optimal error estimates of finite difference methods for the Gross-Pitaevskii equation with angular momentum rotation, Math. Comp., 82 (2013), pp. 99-128.

[3] W. BAO, Y. CAI, Uniform error estimates of finite difference methods for the nonlinear Schrödinger equation with wave operator, SIAM J. Numer. Anal., 50 (2012), pp. 492-521.

[4] W. BAO, Y. CAI, Uniform and optimal error estimates of an exponential wave integrator sine pseudospectral method for the nonlinear Schrödinger equation with wave operator, SIAM J. Numer. Anal., 52 (2014), pp. 1103-1127.

[5] W. BAO, X. Dong, Analysis and comparison of numerical methods for the Klein-Gordon equation in the nonrelativistic limit regime, Numer. Math., 120 (2012), pp. 189-229.

[6] W. BAO, X. Dong AND X. ZhaO, Uniformly accurate multiscale time integrators for highly oscillatory second order differential equations, J. Math. Study, to appear (2014).

[7] W. CAO, B. GuO, Fourier collocation method for solving nonlinear Klein-Gordon equation, J. Comput. Phys., 108 (1993), pp. 296-305.

[8] P. Chartier, N. Crouseilles, M. Lemou and F. Méhats, Uniformly accurate numerical schemes for highly oscillatory Klein-Gordon and nonlinear Schrödinger equations, arXiv: math.NA 1308.0507.

[9] D. Cohen, E. Hairer and C. Lubich, Modulated Fourier expansions of highly oscillatory differential equations, Found. Comput. Math., 3 (2003), pp. 327-345.

[10] D. Cohen, E. Hairer and C. Lubich, Conservation of energy, momentum and actions of numerical discretization of non-linear wave equations, Numer. Math., 110 (2008), pp. 113143.

[11] M. Condon, A. Deaño And A. Iserles, On second order differential equations with highly oscillatory forcing terms, Proc. R. Soc. A, 466 (2010), pp. 1809-1828.

[12] P. Degond, J. Liu And M. Vignal, Analysis of an asymptotic preserving scheme for the EulerPoisson system in the quasineutral limit, SIAM J. Numer. Anal., 46 (2008), pp. 1298-1322.

[13] P. Deuflhard, A study of extrapolation methods based on multistep schemes without parasitic solutions, ZAMP., 30 (1979), pp. 177-189.

[14] D. B. Duncan, Symplectic finite difference approximations of the nonlinear Klein-Gordon equation, SIAM J. Numer. Anal., 34 (1997), pp. 1742-1760.

[15] E. FaOU, K. Schratz, Asympotic preserving schemes for the Klein-Gordon equation in the nonrelativistic limit regime, Numer. Math., 126 (2014), pp. 441-469.

[16] B. Garcia-Archilla, J. M. Sanz-Serna and R. D. Skeel, Long-time-step methods for oscillatory differential equations, SIAM J. Sci. Comput., 20 (1998), pp. 930-963.

[17] W. GaUtSCHI, Numerical integration of ordinary differential equations based on trigonometric polynomials, Numer. Math., 3 (1961), pp. 381-397.

[18] J. Ginibre, G. Velo, The global Cauchy problem for the nonlinear Klein-Gordon equation, Math. Z., 189 (1985), pp. 487-505.

[19] J. Ginibre, G. Velo, The global Cauchy problem for the nonlinear Klein-Gordon equation-II, Ann. Inst. H. Poincaré Anal. Non Linéaire, 6 (1989), pp. 15-35.

[20] R. Glassey, On the asymptotic behavior of nonlinear wave equations. Trans. Am. Math. Soc. 182 (1973), pp.187-200.

[21] R. Glassey, M. Tsutsumi, On uniqueness of weak solutions to semi-linear wave equations. Commun. Partial Differ. Eqn., 7 (1982), pp. 153-195.

[22] V. Grimm, M. Hochbruck, Error analysis of exponential integrators for oscillatory secondorder differential equations, J. Phys .A: Math. Gen., 39 (2006), pp. 5495.

[23] M. Hochbruck, Сн. Lubich, A Gautschi-type method for oscillatory second-order differential equations, Numer. Math., 83 (1999), pp. 402-426.

[24] E. Hairer, Ch. Lubich and G. Wanner, Geometric Numerical Integration: StructurePreserving Algorithms for Ordinary Differential Equations, Springer, Berlin, 2006.

[25] S. Jin, Efficient Asymptotic-Preserving (AP) schemes for some multiscale kinetic equations, SIAM J. Sci. Comp., 21 (1999), pp. 441-454.

[26] S. JimÉnez, L. VÁzQuez, Analysis of four numerical schemes for a nonlinear Klein-Gordon equation, Appl. Math. Comput., 35 (1990), pp. 61-94.

[27] B. Leimkuhler, S. Reich, Simulating Hamiltonian Dynamics, Cambridge University Press, 
2004.

[28] S. Machinara, The nonrelativistic limit of the nonlinear Klein-Gordon equation, Funkcial. Ekvac., 44 (2001), pp. 243-252.

[29] S. Machinara, K. NAKanishi and T. Ozawa, Nonrelativistic limit in the energy space for nonlinear Klein-Gordon equations, Math. Ann., 322 (2002), pp. 603-621.

[30] N. Masmoudi, K. NAKAnishi, From nonlinear Klein-Gordon equation to a system of coupled nonlinear Schrödinger equations, Math. Ann., 324 (2002), pp. 359-389.

[31] C. Morawetz, W. Strauss, Decay and scattering of solutions of a nonlinear relativistic wave equation, Comm. Pure Appl. Math., 25 (1972), pp. 1-31.

[32] B. Najman, The nonrelativistic limit of the nonlinear Klein-Gordon equation, Nonlinear Anal., 15 (1990), pp. 217-228.

[33] H. PeCheR, Nonlinear small data scattering for the wave and Klein-Gordon equation, Math. Z., 185 (1984), pp. 261-270.

[34] J. J. SAKURAI, Advanced Quantum Mechanics, Addison Wesley, New York, 1967.

[35] J. M. SAnZ-Serna, Mollified impulse methods for highly oscillatory differential equations, SIAM J. Numer. Anal., 46 (2008), pp. 1040-1059.

[36] I. E. SEgaL, The global Cauchy problem for a relativistic scalar field with power interaction, Bull. Soc. Math. Fr., 91 (1963), pp. 129-135.

[37] J. C. H. Simon, E. TAflin, The Cauchy problem for non-linear Klein-Gordon equations, Commun. Math. Phys., 152 (1993), pp. 433-478.

[38] J. Shen, T. Tang And L. Wang, Spectral Methods: Algorithms, Analysis and Applications, Springer-Verlag, Berlin Heidelberg, 2011.

[39] W. Strauss, L. VÁzquez, Numerical solution of a nonlinear Klein-Gordon equation, J. Comput. Phys., 28 (1978), 271-278.

[40] T. TAO, Local and Global Analysis of Nonlinear Dispersive and Wave Equations, CBMS Regional Series In Mathematics, 2006.

[41] M. Tsutsumi, Nonrelativistic approximation of nonlinear Klein-Gordon equations in two space dimensions, Nonlinear Anal., 8 (1984), pp. 637-643. 